\title{
„Globales Prekariat"? Ungleiche und kombinierte Informalisierung als sozialwissenschaftliche und politische Herausforderung
}

\author{
Bert Bach $^{\star}$ und Karola Blaha ${ }^{* *}$
}

\section{Zusammenfassung}

Bach/Blaha versuchen in ihrem Beitrag eine globale Einordnung von prekären Arbeitsverhältnissen in konzeptueller und empirischer Hinsicht. Ausgangspunkt ist die seit den 199oer-Jahren intensiver geführte Prekarisierungsdebatte, die sich auf institutionelle Kontexte des globalen Nordens bezieht. Mit Verweis auf die schon länger zurückreichende und auf breitere, vielfältigere Phänomene im globalen Süden fokussierende Diskussion $z u$ informeller Beschäftigung und Informalisierung präferieren die AutorInnen den Begriff der Informalisierung als übergeordneten, global verwendbaren bzw. weniger kontextabhängigen Begriff, um Phänomene der Unsicherheit und Verwundbarkeit sowie Verbindungen zwischen formalen und informellen Beschäftigungssegmenten etwa im Rahmen transnationaler Wertschöpfungsketten zu fassen. Vor dem Hintergrund einer sehr eingeschränkten Datenlage unternimmt der Beitrag den Versuch einer Quantifizierung verschiedener Beschäftigungsformen im globalen Rahmen, um sich einer globalen Klassenstruktur anzunähern. Der zweite Teil des Beitrags schließt an diese empirischen Befunde an und geht der Frage nach, welche Herausforderungen für die Organisation und Artikulation von Interessen von informell Beschäftigten sowie von subaltern Arbeitenden generell damit verbunden sind. Mit Bezugnahme auf Machtressourcenansätze werden Überlegungen zu Möglichkeiten und Barrieren der Organisierung angestellt.

Schlagwörter: Prekarisierung; prekär; Prekariat; Informalisierung; informelle Beschäftigung; Klassenstruktur; global; Organisierung; Gewerkschaften; Machtressourcen

\section{"Global Precariat"? Unequal and Combined Informalization as a challenge for research and political action}

\section{Abstract}

In their contribution, Bach/Blaha attempt to classify precarious employment relationships globally in conceptual and empirical terms. The starting point is the precarization debate, which has been conducted more intensively since the 1990 and refers to institutional contexts of the global North. With reference to the discussion on informal employment and informalization in the global South, which goes back some time and focuses on broader, more diverse phenomena, the authors prefer the term informalization as a superordinate, globally usable or less context-dependent term in order to grasp phenomena of insecurity and vulnerability as well as connections between formal and informal employment segments, for example within the framework of transnational value chains. Against the background of very limited data, this paper attempts to quantify different forms of employment in a global context in order to approach a global class structure. The second part of the article follows on from these empirical findings and examines challenges for the organisation and articulation of interests of informal workers and subaltern workers in general. With reference to power resource approaches, considerations are made about opportunities and barriers of organizing.

Keywords: Precarity; precarious; precarization; informalization; informal employment; class structure; global; organizing; trade unions; social movement unionism; power resources

\footnotetext{
* Kontaktperson Bert Bach. E-Mail: bert.bach@aon.at, Soziologe in Wien

** Ökonomin in Wien
} 


\section{Einleitung}

Inspiriert von den Debatten über das Konzept des „Prekariats" von Guy Standing im Global Labour Journal (Standing 2016b), wollen wir uns der Frage widmen, ob es sinnvoll ist, von einem "globalen Prekariat“ zu sprechen und welche analytischen und politischen Implikationen damit verbunden sein könnten.

Sowohl Standing als auch Bourdieu als einer der wichtigsten Referenzautoren späterer Prekarisierungsdebatten haben betont, dass ihre Auseinandersetzungen mit der Prekarisierung auf Forschungsprojekten und Beobachtungen zu Phänomenen im globalen Süden basieren. Dennoch sind die weiteren Diskussionen, wie verschiedene im Global Labour Journal veröffentlichte Artikel zeigen, deutlich von regionalen Besonderheiten des globalen Nordens geprägt, die Verallgemeinerungen erschweren.

Während Standing in seinen beiden Büchern (2011; 2014) qualitative Aspekte auflistet, um prekäre Situationen und Karrieren $\mathrm{zu}$ charakterisieren und daraus politische Handlungsoptionen abzuleiten (vgl. $\mathrm{zu}$ diesen Handlungsoptionen und der Kritik an der Top-down-Orientierung Paret 2016a), wollen wir zwei Aspekte in die Diskussion einbringen: Durch einen ersten quantitativen Ansatz, der auf verfügbaren Sekundärdaten basiert, versuchen wir, weitere Debatten über konzeptionelle Abgrenzungen und Verfeinerungen anzuregen, die sich daraus ergeben, und die Frage zu stellen, welche Implikationen für weitere Diskussionen durch die Berücksichtigung dieser Proportionen (auch wenn Lohnarbeit global gesehen zunimmt, ist das sogenannte Normalarbeitsverhältnis ein Minderheitenprogramm, dem nur in den Hocheinkommensländern eine gewisse empirische Relevanz zukommt) berührt werden. Konkrete Formen der Organisation subalterner ArbeiterInnen sind der zweite Schwerpunkt dieses Papiers. Berücksichtigt man, dass für den globalen Norden typische Organisations- und Arbeitskampfformen nur eine Teilmenge vieler möglicher Formen und aus globaler Sicht empirisch minoritär sind, wirft dies eine Reihe von Fragen auf, z. B. nach dem gewerkschaftlichen Selbstverständnis etwa im Hinblick auf die Organisierung von informell Beschäftigten oder dem Verhältnis zwischen Parteien, Gewerkschaften, NGOs und sozialen Bewegungen, die aus unserer Sicht nicht so einfach oder dichotom zu beantworten sind, wie es etwa bei Guy Standing anklingt.

Unser Beitrag ist auch ein Versuch, die sehr informative und interessante Debatte über die Prekarität und das „Prekariat“ durch Anregungen und Fragen $\mathrm{zu}$ erweitern und weitere Beiträge anzuregen. Dabei schlagen wir vor, den Begriff der Informalität bzw. Informalisierung als die übergeordnete Kategorie für die sozialwissenschaftliche Analyse und politische Schlussfolgerungen zu wählen. Von dieser Warte aus betrachtet soll den verschiedenen Spielarten des Prekären, der Unsicherheit, der Marginalität und Abhängigkeit jeglicher Formen von Arbeit nachgegangen und Fragen der Organisierungsfähigkeit erörtert werden. Diese Aspekte standen schließlich auch im Zentrum, als Marx von Proletariat und Übervölkerung gesprochen hat.

Der Beitrag ist wie folgt aufgebaut: Im Kapitel 2 wird der Prekaritätsdiskurs eingeordnet in die Debatten über informelle Arbeitsformen, die in Hochlohnländern anders als in Ländern mit mittleren und niedrigen Einkommen geführt werden. Kapitel 3 liefert einen ersten, recht groben Eindruck von globalen Klassenstrukturen oder genauer: Schichten von Arbeit, Einkommen und sozialer Absicherung. Kapitel 4 geht Fragen der Formierung und Organisierung subalterner ArbeiterInnen nach. Kapitel 5 versucht schließlich zu Schlussfolgerungen zu kommen. Begrifflich werden wir das zu eng an den Erfahrungen von westlichen Industrieländern orientierte Konzept des Prekariats ebenso hinter uns lassen wie den älteren Begriff des Proletariats, indem wir uns an den Überlegungen zu den subalternen ArbeiterInnen von Marcel van der Linden orientieren. Dieses Konzept stellt die erzwungene Kommodifizierung von Arbeit, also die Heteronomie von Arbeit, in den Mittelpunkt.

\section{Verortung des Prekaritätsdiskurses}

„What you are calling precarious work sounds like what we in Ghana call - work."

Ohne in diesem Beitrag näher auf eine Genealogie des Prekaritätsdiskurses eingehen zu können (vgl. etwa Candeias 2008), wird an dieser Stelle darauf verwiesen, dass das Prekaritätskonzept, das seit den 1990er-Jahren verstärkte sozialwissenschaftliche und politische Aufmerksamkeit genießt, spätestens seit den 1970erJahren in unterschiedlichen Ländern (v. a. Italien und Frankreich) und Zusammenhängen und - ähnlich wie der Begriff Klasse - sowohl in analytischer als auch in

1 Reaktion eines Forschers aus Ghana auf eine Präsentation der International Labour Organization (ILO) im Rahmen einer Konferenz in Südafrika (zitiert nach Mosoeta/ Stillerman/Tilly 2016). 
politischer Weise verwendet wurde. Der Begriff soll eine Entwicklung beschreiben und sichtbar machen, diese aber gleichzeitig als politisch forcierte kritisieren und eine Referenzfolie für politische Handlungsmöglichkeiten bieten.

Grob zusammengefasst geht es darum, neue bzw. wiederkehrende Formen von Unsicherheit im globalen Norden am Ende des 20. Jahrhunderts begrifflich zu fassen. Castel (2000) verortet aktuelle Formen von Prekarität in seiner Genealogie lohnarbeitsbezogener Integrationsformen in Abgrenzung zu anderen zeitdiagnostisch verwendeten Konzepten und Thesen wie Exklusion oder einer sich verallgemeinernden Pauperisierung eher an den Rändern der (unteren) Mitte. Die Zone der Verwundbarkeit unterscheidet sich dadurch von der von ihm so bezeichneten Zone der Entkopplung, dass Prekarisierungserfahrungen als Formen der Unsicherheit und Verunsicherung nicht mit Armut oder Exklusion zusammenfallen, sondern gesellschaftlich breiter streuen und Prekarisierungstendenzen auch auf die Zone der Integration ausstrahlen, indem sie eine disziplinierende Wirkung entfalten (Dörre 2013). Die Zone der Verwundbarkeit ist sozialstrukturell und im Hinblick auf normative Erwartungen näher am Zentrum angesiedelt und nur relational $\mathrm{zu}$ diesem Zentrum $\mathrm{zu}$ definieren. Es geht also bei der nördlichen bzw. westlichen Prekarisierungsdebatte - verkürzt gesprochen - um Formen der postsozialstaatlichen Desintegration und um Legitimationsprobleme, die tendenziell in der Mitte der Gesellschaft Platz finden. Vor der Referenzfolie fordistischer Wohlfahrtsstaatlichkeit wird ein neues Disziplinarregime konstatiert, das bereits erreichte Standards der Absicherung und Teilhabe unterläuft und reduziert und Unsicherheit verallgemeinert. Wichtig ist in diesem Zusammenhang, dass es sich bei Prekarität um eine relationale Kategorie handelt, „deren Aussagekraft wesentlich von der Definition gesellschaftlicher Normalitätsstandards abhängt" (Dörre 2013: 33). Von diesem Ausgangspunkt stellt sich für uns die Frage: Was passiert mit dem Prekaritätskonzept, wenn man den Bezugsrahmen geografisch erweitert?

In den vergangenen Jahren wurde versucht, die vorher unverbundenen Diskurse zu Prekarität bzw. Prekarisierung, die theoretisch und empirisch vor allem für den globalen Norden plausibilisiert wurden (vgl. etwa Castel/Dörre 2009), mit Phänomenen der Marginalität, der Informalität bzw. Informalisierung, wie sie schon seit Jahrzehnten vor allem mit Bezug auf den globalen Süden diskutiert werden (Munck 2013), zu verknüpfen bzw. die unterschiedlichen Konzepte auf Ähnlichkeiten und Unterschiede sowie auf Grenzen der Übertragbarkeit hin zu untersuchen (vgl. etwa Burchardt/Peters/ Weinmann 2013; Mayer-Ahuja 2017). Dabei geraten Ähnlichkeiten und Schnittmengen, aber auch gravierende Unterschiede in den Blick. ${ }^{2}$

Wenn ein bestimmtes Set an institutioneller Einhegung des Kapitalismus (Normalarbeitsverhältnis, Wohlfahrtsstaat) als Referenzfolie für die im Prekarisierungsdiskurs konstatierten Erosionsprozesse herangezogen wird, wirft das Konzept bei einer Anwendung auf periphere und semi-periphere Weltregionen große Probleme auf: „Verengt man Prekarität auf ihre postsozialstaatlichen Ausprägungen in fortgeschrittenen Kapitalismen, so wäre dieses Phänomen in den Gesellschaften des globalen Südens gar nicht existent" (Dörre 2013: 39). Das Goldene Zeitalter des nördlichen Fordismus (ca. 1950-1973) war sicher kein goldenes für die damaligen Kolonien (aber auch nicht für Frauen und MigrantInnen im globalen Norden). Andererseits wurden im globalen Süden für diese Länder wichtige Elemente sozialstaatlicher Absicherung erst in einem Zeitabschnitt erreicht, der im Norden mit neoliberaler Deregulierung und Sozialabbau assoziiert wird (Scully 2016). Auch die von T. H. Marshall angenommenen Zusammenhänge von „Citizenship and Social Class" sowie die im Norden beobachtete lineare Abfolge von bürgerlichen, politischen und sozialen Rechten treffen so auf den globalen Süden nicht zu. ${ }^{3}$ Während sich im Norden nach dem Erreichen politischer Teilhaberechte institutionalisierte

2 Eine Ausgabe des Global Labour Journals (2016/17) widmet sich dem Prekaritätskonzept von Standing (2015, 2016a) sowie verschiedenen Arten der Kritik daran, die vor allem auf die zeitliche und räumliche Begrenztheit der zugrundeliegenden Annahmen abzielt und deren Verallgemeinerbarkeit problematisiert. Auf die einzelnen Diskussionsstränge und vorgebrachten Argumente kann in diesem Beitrag nur kursorisch eingegangen werden (Scully 2016, Paret 2016a; Paret 2016b; Braga 2016; Standing 2015a). Auch in der Zeitschrift International Labor and Working-Class History wurde eine Debatte zu Prekarität in globaler Perspektive geführt (vgl. einleitend Mosoetsa/Stillerman/Tilly 2016). Umgekehrt wird auch die Bezugnahme auf Beobachtungen und Erkenntnisse, die im globalen Süden gewonnen wurden, für Prozesse der Informalisierung im globalen Norden sowie Wechselwirkungen zwischen Informalisierungsprozessen in verschiedenen Weltregionen diskutiert (vgl. etwa Parnreiter 1997; Sassen 1997).

3 Standing (2015b, 2016) spricht im Zusammenhang mit dem von ihm als „neue Klasse im Werden“ verstandenen Prekariat von „denizens“ im Unterschied zu „citizens“. Prekarisierung impliziert demnach eine Art „Unterschichtung“, das Prekariat fällt als „BürgerInnen zweiter Klasse“ hinter bereits erreichte Standards von Rechtsansprüchen, die für die Mehrheitsgesellschaft gelten, zurück. 
Formen des Klassenkompromisses (Sozialpartnerschaft) herausbildeten und soziale Rechte ausgebaut wurden, war das Entwicklungsversprechen in vielen südlichen postkolonialen Staaten nicht in dieser Weise mit ausgedehnten politischen oder sozialen Rechten, sondern häufiger mit paternalistischen und klientelistischen Formen der Einbindung von oben verbunden:

„Where the industrial working class was a minority, southern class compromise was essentially a bargain between the developmentalist state and the urban poor (shanty dwellers, unemployed youths, street vendors, and various disaffected social groups), not the organized associations of capital and labor. Public assistance was provided in exchange for political loyalty. "(Lee/Kofman 2012: 394)

Der für diesen Artikel wichtigste Einwand betrifft die Rolle des Normalarbeitsverhältnisses als regulative Idee bzw. analytisches und normatives Zentrum des Prekarisierungsdiskurses. Zwar spielte die formelle Beschäftigung auch in Ländern wie Indien eine wichtige Rolle als normatives Ideal (Mayer-Ahuja 2017), war aber dort empirisch immer eine minoritäre Form der Erwerbsarbeit (maximal 10 Prozent, vor allem im öffentlichen Sektor, vgl. etwa Ahuja 2013), die im Zuge neoliberaler Restrukturierungen im Hinblick auf ihre empirische Verbreitung an Bedeutung verloren hat.

Auf die inzwischen weit verzweigte Debatte zum informellen Sektor bzw. zu informeller Beschäftigung kann im Rahmen dieses Beitrags nicht vertiefend eingegangen werden. Der Begriff informeller Sektor wurde in den 1970er-Jahren im Umfeld der International Labour Organization (ILO) im Zusammenhang mit Studien zu Ghana und Kenia geprägt (ILO 1972, Hart 1973) und spielte - mit zum Teil sehr unterschiedlichen Prämissen und Schlussfolgerungen - seither eine wichtige Rolle in modernisierungstheoretischen, dependenztheoretischen und neoliberalen Diskussionssträngen zu abhängiger Entwicklung bzw. peripherem Kapitalismus. Die dualistische Konzeption zweier voneinander getrennter Sektoren wurde bald kritisiert (Breman 1976), inzwischen wird von einem Kontinuum und vielfältigen Verbindungslinien zwischen formellen und informellen Aktivitäten ausgegangen: ${ }^{4}$ „,Fliegende ${ }^{6}$ Straßenhänd-

4 Das gilt nicht nur für Arbeits-, sondern auch für Wohnformen, wie etwa Mike Davis (2007) anhand einer Typologie von Slums aufzeigt. Auch hier gilt: Eigentumsoder Mietwohnungen mit Vertrag und die illegale Besetzung und Bebauung von Land stellen zwei Pole dar, dazwischen gibt es eine Vielzahl an Mischformen, Überlappungen und Grauzonen, die mit unterschiedlichen Graden der Abhängigkeit und Unsicherheit einhergehen. ler in Cali vertreiben Produkte großer Industrien [...] und informelle handwerkliche Betriebe in Bogotá produzieren Schuhe für Unternehmen des formellen Sektors" (Parnreiter 1997: 203). Informelle Tätigkeiten sind oft Bestandteile transnationaler Wertschöpfungsketten: „Elektronikkonzerne lassen über eine Kette von Subunternehmern in informellen Werkstätten und in Heimarbeit in Mexico City produzieren [...] und US-Bekleidungsfirmen vergeben - wiederum über Zwischenhändler - Näharbeiten an kleinere Schneidereibetriebe in Guatemala" (ebd.). Auch in der Textilindustrie in Asien oder in der Maquiladora-Montage in Lateinamerika spielen informell produzierte Vor- und Zwischenprodukte eine wichtige Rolle (Langthaler 1997; Weinmann/Burchardt 2013).

Eine Erweiterung des Blickwinkels auf das Phänomen Prekarität/Prekarisierung in Richtung "globales Prekariat" bedeutet daher, sich des jeweiligen Referenzrahmens zu vergewissern, von dem aus prekäre Positionen und Dynamiken beobachtet werden. Die konkreten Normalitätsstandards sind global sehr unterschiedlich, es ist von einer großen Bandbreite an verschiedenen Arten von Verwundbarkeit und Unsicherheit auszugehen. Schon für einen ersten Schritt der deskriptiven Verortung prekärer Lebenslagen sind daher Konzepte zu verwenden, die nicht stark an eine spezifische institutionelle Konfiguration gebunden sind. Aus unserer Sicht ist Informalität bzw. Informalisierung ein übergeordneter Begriff, mit dem sowohl Beschäftigungsformen, die für den globalen Süden typisch sind, als auch verschiedene Formen der Entstandardisierung von Arbeitsverhältnissen im globalen Norden erfasst werden können. Anstatt den Begriff der Prekarisierung zu verallgemeinern und für eine globale Betrachtung zu verwenden, schlagen wir daher vor, von ungleicher und kombinierter Informalisierung zu sprechen und bei allen Unterschieden auch darauf zu achten, dass die verschiedenen Elemente und Dynamiken dieses Prozesses im Rahmen einer kapitalistischen Weltökonomie stattfinden.

Ungleiche und kombinierte Informalisierung bezeichnet eine Entwicklung, die sowohl im globalen Norden als auch im globalen Süden zu beobachten ist, allerdings in unterschiedlichen Formen und auf unterschiedlichen Niveaus. Der Begriff betont auch, dass diese Prozesse miteinander verbunden sind. So wie innerhalb eines Betriebs im globalen Norden der Einsatz von Leiharbeit Druck auf Stammbelegschaften und deren „Normalarbeitsverhältnis“ ausübt, so sind auch formelle Beschäftigungsformen, formelle und infor- 
melle Beschäftigung sowie verschiedene Formen informeller Beschäftigung in transnationale Austausch- und Konkurrenzbeziehungen eingebunden. Die Forcierung informeller Beschäftigung an verschiedenen Punkten von transnationalen Wertschöpfungsketten stellt eine wichtige Option für Kapitalstrategien im Hinblick auf Kostensenkung und Flexibilisierung bzw. Profitsteigerung dar: „Informalisierung ist so gesehen einer der möglichen Auswege aus der Krise des ,fordistischen Massenproduktionssystems. Ein anderer ist die Verlagerung der Produktion in Niedriglohnländer, ein dritter der vermehrte Zukauf von Produkten oder Komponenten, die in der (Semi)peripherie hergestellt wurden“ (Parnreiter 1997: 212).

Sassen (1997) verweist am Beispiel von New York auf verschiedene Formen und Funktionen der verstärkten Informalisierung, die mit Entwicklungen wie Polarisierung der Einkommen und - damit verbunden - der Nachfrage nach entsprechenden Dienstleistungen einhergehen: „Es gibt zahlreiche Beispiele dafür, wie die wachsende Einkommensungleichheit die Konsumstruktur verändert und wie dieser Umstand wiederum auf die Arbeitsorganisation zurückwirkt" (Sassen 1997: 241). In Summe ergibt sich ein „Steigerungsspiel“, das von verschiedenen Akteuren auf unterschiedlichen Ebenen vorangetrieben wird: „Informalisierung ergibt sich als ein Komplex von flexibilitätsmaximierenden Strategien, zu denen Individuen und Firmen, Konsumenten und Produzenten in einem Kontext sich verschärfender Ungleichheit von Einkommen und Rentabilität Zuflucht nehmen“" (Sassen 1997: 247).

Neuere Studien verweisen auf verschiedene Segmente und hierarchische Gliederungen innerhalb der informellen Ökonomie, auch die ILO versucht mittlerweile, Perspektiven der verschiedenen Ansätze zu integrieren (ILO 2018a; wir orientieren uns in unserer Darstellung im nachfolgenden Kapitel „Globale Klassenstrukturen“ an Konzepten und Daten der ILO). Die einzelnen Segmente können sich deutlich etwa im Hinblick auf ungleiche Möglichkeiten der Einkommensgenerierung oder der Segregation nach Geschlecht oder ethnischer Herkunft unterscheiden (Chen 2013). Aus unserer Sicht ist es bei einer Analyse informeller Erwerbstätigkeit wesentlich, verkürzte Sichtweisen, die informell mit unreguliert gleichsetzen und als Problemlösung politische Regulierung nahelegen, $\mathrm{zu}$ vermeiden. So wie neoliberale Wettbewerbsstaaten nicht mit völlig deregulierten Nachtwächterstaaten gleichzusetzen sind, weil die Eingriffstiefe nicht generell zurückgefahren, sondern vor allem Art und Berei- che der Intervention verändert werden, so wäre auch im Hinblick auf informelle Beschäftigung darauf zu achten, wie Informalität politisch hergestellt, unterstützt oder forciert wird, etwa in Form bewusster selektiver Auslassungen einzelner Bereiche oder in Form der Einrichtung von Sonderwirtschaftszonen, die in arbeitsrechtlicher Hinsicht als extraterritoriale Zonen eingerichtet werden (Altvater/Mahnkopf 2002; Lee/ Kofman 2012; Carstensen 2014).

Eine globale Erweiterung des Blickwinkels auf Erwerbsarbeit führt auch dazu, dass Phänomene wie Kinderarbeit oder verschiedene Formen von unfreier Arbeit in den Blick geraten, die in europäischen Debatten bisher nur am Rand und als Extremfälle der globalen Erwerbslandschaft diskutiert werden, nicht aber als deren konstitutiver Bestandteil.

Eine Horizonterweiterung über die OECD-Welt hinaus zeigt, dass der globalisierte Kapitalismus nicht mit einer allgemeinen Proletarisierung im Sinne einer Verallgemeinerung der formellen Lohnarbeit einhergeht. Zwar führen Prozesse der Inwertsetzung und (Re-)Kommodifizierung zu einer Art passiver Proletarisierung, indem traditionelle Lebensweisen jenseits der kapitalistischen Weltwirtschaft tendenziell verunmöglicht werden. Das heißt aber nicht, dass sich Lohnarbeit bzw. formelle Erwerbsarbeit (schon gar nicht ein „Normalarbeitsverhältnis“ im nordwesteuropäischen Sinn) verallgemeinert und die gesellschaftliche Gesamtarbeiterin sozusagen aktiv im Sinne von Marx (Äquivalententausch mittels Arbeitsvertrag, Mehrwert produzierende Lohnarbeit) proletarisiert wird (Kössler 2016). Verstärkte Abhängigkeit von Geld und Markteinkommen ist nicht gleichzusetzen mit Lohnarbeit: „We must insist that ,proletarian' is not a synonym for ,wage labourer', but for dispossession, expropriation and radical dependence on the market" (Denning 2010: $81)$.

Die in diesem Rahmen nur kursorisch mögliche Übersicht über Grenzen des Prekarisierungskonzepts zeigt, dass bei einer globalen Betrachtungsweise Konzepte notwendig sind, die unabhängiger von spezifischen Entwicklungen und institutionellen Kontexten im globalen Norden sind und eine Annäherung an vielschichtige Erwerbsformen ermöglichen bzw. nicht Phänomene ausblenden oder als Extremfälle begrifflich an den Rand drängen, die global gesehen eher die Norm als die Ausnahme darstellen. Im Folgenden wird es daher darum gehen, anhand eines begrifflichen Grundgerüsts verfügbare Daten in einer Weise zu gruppieren, die eine Annäherung an eine globale 
Klassenstruktur zumindest in groben Größenordnungen ermöglicht. Aufgrund der eingeschränkten Datenverfügbarkeit ist nur eine Annäherung über den Arbeitsmarktstatus möglich. Eine halbwegs adäquate Abbildung von prekären Positionen würde wesentlich mehr und feinkörnigere Daten auf Individual- und Haushaltsebene erfordern, um zumindest die finanzielle Situation und die soziale Absicherung im Zeitverlauf, d. h. die Häufigkeit und Persistenz von prekären Episoden, zu identifizieren. Das ist bisher nur in einzelnen Ländern mit hervorragender Datenbasis möglich (vgl. etwa Allmendinger et al. 2018).

\section{Globale „Klassenstrukturen” - Schichten von Arbeit, Absicherung und Einkommen}

Im vorliegenden Beitrag wird, in Abweichung bzw. Weiterentwicklung des marxistischen Begriffes des Proletariats, mit den Termini subalterne ArbeiterInnen (Marcel van der Linden ${ }^{5}$ ) und relative Überbevölkerung (Karl Marx, Srnicek/Williams ${ }^{6}$ ) gearbeitet. Die engen klassentheoretischen Begriffe Bourgeoisie-Proletariat, die anknüpfen an der Mehrwertproduktion bzw. an

5 In seinem Plädoyer für die Neubestimmung der Welt-Arbeiterklasse nennt Marcel van der Linden (2010) die Heteronomie als das Gemeinsame der „subalternen ArbeiterInnen“, das Eigentum an Produktionsmitteln ist dabei weniger wichtig. Diese Subalternen sind „freie“ LohnarbeiterInnen, Selbstständige, Sklaven und Lumpenproletarier, deren Arbeitskraft hat in unterschiedlicher Weise Warencharakter angenommen. Er definiert demzufolge subalterne Arbeit als ,jeder Träger der Arbeitskraft, dessen Arbeitskraft unter ökonomischem oder nicht-ökonomischem Zwang an einen anderen verkauft oder vermietet wird, gehört zu den subalternen Arbeitern, und zwar ungeachtet dessen, ob der Träger selbst der Verkäufer oder Vermieter ist, und ungeachtet dessen, ob er auch selbst über Produktionsmittel verfügt" (van der Linden 2010: 375).

6 In Anlehnung an Marx (der flüssige, latente und stockende Formen der Überbevölkerung unterschied; siehe Marx, 1867/1989, 670) definieren Srnicek/Williams (2014) die „relative Überbevölkerung“ als die, die außerhalb der Lohnarbeit unter kapitalistischen Produktionsbedingungen stehen; sie unterscheiden vier Typen: kapitalistisch (Arbeitslose und Unterbeschäftigte); nicht-kapitalistisch (neue Formen der Subsistenzwirtschaft an den Rändern des Kapitalismus); latent (vorkapitalistische ökonomische Formationen, Reservoir protoproletarischer kleinbäuerlicher Bevölkerung, unbezahlte Hausangestellte, dequalifizierte Honorarkräfte in freien Berufen) und inaktiv (Entmutigte, StudentInnen, Invalide etc.); nach dieser Einteilung gehört der Großteil des informellen Sektors (weil keine kapitalistische Akkumulation vorherrscht) zur Überschussbevölkerung. der Verfügung über Produktionsmittel, scheinen nicht hinreichend geeignet für eine Analyse auf globaler Ebene, weil die klassische Lohnarbeit nur einen Teil der gesamten globalen Arbeit, die in der einen oder anderen Form angeboten wird, ausmacht. Dominant, im empirischen Sinn, sind die Randbereiche und Übergangspositionen der kapitalistischen Produktion und Akkumulation, die Kernbereiche (im klassischen marxistischen Sinn) kommen hingegen vor allem in Hocheinkommensländern vor; aber auch hier greifen Informalisierungstendenzen mehr und mehr um sich, die mit der Zunahme von atypischen Beschäftigungsverhältnissen einhergehen.

Die Analyse von globalen Klassenstrukturen sieht sich mit erheblichen Problemen in Bezug auf die Verfügbarkeit von Daten konfrontiert. Über die Stellung im Produktionsprozess, die Verfügungsmacht über Produktionsmittel, die unterschiedlichsten Formen von subalterner Arbeit etc. liegen nur vereinzelte Informationen für einzelne Länder meist in den wohlhabenderen Regionen des Nordens vor. Auch lassen sich verschiedene Konzepte (wie etwa jenes der Lohnarbeit, der Arbeitslosigkeit, des Prekariats, der stillen Reserve etc.) nicht einfach von einer Weltgegend auf eine andere übertragen. Der Fokus der nachfolgenden Analyse soll auf vier Ländergruppen liegen, weil damit sowohl die doch recht ungenaue Begrifflichkeit globaler Norden - globaler Süden wie auch eine ausufernde Darstellung auf Ebene einzelner Länder vermieden werden: die vier Gruppen sind Länder mit hohem Bruttonationaleinkommen (BNE), mit mittleren (hier werden zwei Gruppen unterschieden) und mit niedrigen Einkommen. ${ }^{7}$ Analysiert wird die Verteilung der Bevölkerung auf verschiedene Statuspositionen (Lohnarbeit, prekäre Beschäftigung, Kapital, Inaktivität, Unterbeschäftigung, Überschussbevölkerung) bzw. ihre Veränderung über die Zeit, die Verteilung nach globalen Einkommensklassen, nach informeller Arbeit und sozialer Absicherung.

Im Zeitraum 1992-2017 stieg die Weltbevölkerung von ca. 5,5 Mrd. auf ca. 7,5 Mrd. Personen an, das globale Arbeitsangebot (Lohnarbeit, Kapital,

7 Die Grenzen im Bruttonationaleinkommen (BNE) liegen bei: Niedrige Einkommensländer - kleiner USD 1.005 pro Kopf; mittlere Einkommen/niedrig - zwischen USD 1.006 und 4.035; mittlere Einkommen/hoch - zwischen USD 4.036 und USD 12.234; Hocheinkommensländer - über USD 12.235 . 
Abbildung 1: Weltbevölkerung, globale Lohnarbeit, Prekariat, Überschussbevölkerung (in \% der Weltbevölkerung, $1992=5,46 \mathrm{Mrd}$., $2017=7,53 \mathrm{Mrd}$.)

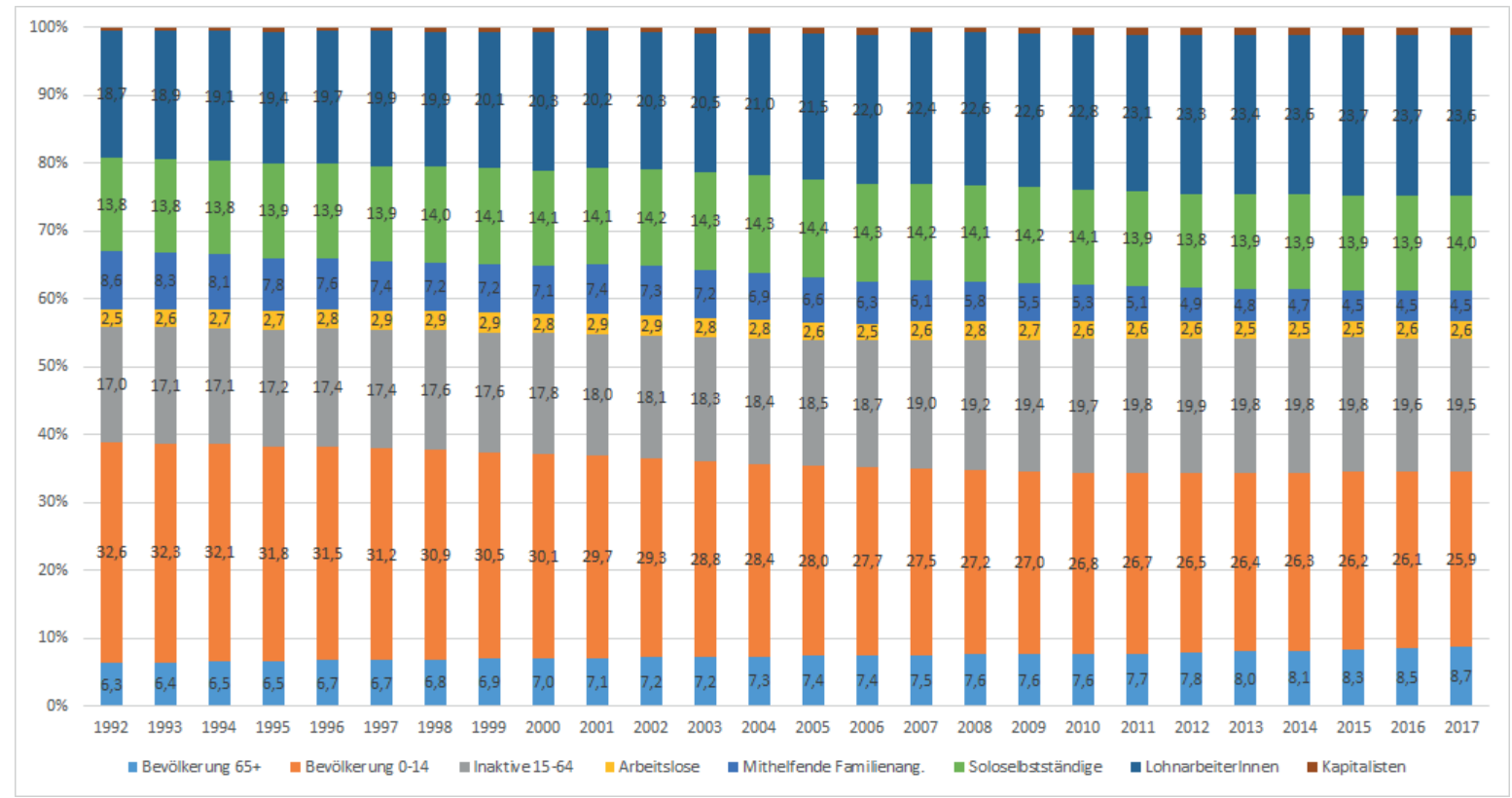

Quelle: ILO-WESO, Weltbank WDI

Arbeitslose) $)^{8}$ erhöhte sich lt. ILO von etwa 2,4 Mrd. auf etwa 3,5 Mrd. Menschen. Der Betrachtungszeitraum wird gewählt einerseits aus Gründen der Datenverfügbarkeit, aber auch, weil sich durch den Eintritt der ehemals kommunistischen Staaten und von China und Indien in den Weltmarkt die globalen Relationen von Lohnarbeit und Kapital deutlich zuungunsten der Subalternen verändert haben.

\section{a) Subalterne ArbeiterInnen: Lohnarbeit - prekäre Beschäftigungsformen - Überschussbe- völkerung}

Abbildung 1 zeigt die Entwicklung der prozentuellen Verteilung der gesamten Weltbevölkerung im Zeitraum 1992-2017 auf verschiedene Statuspositionen. Diese Darstellung hat den Vorteil, dass sie die gesamte Bevölkerung in den Blick nimmt: Während in Hochein-

8 Zur Definition der Begriffe Lohnarbeit („employee“ - bezahlte Arbeit mit einem Arbeitsvertrag, Entlohnung unabhängig vom Umsatz), Kapital („employer"), „gefährdete“ Beschäftigung („vulnerable employment“), Arbeitsangebot („labour force“), inaktive Personen („not in the labour force") etc. siehe: https://www.ilo.org/wesodata/definitionsand-metadata. Zu den Konzepten der Arbeit („work“), der Beschäftigung („employment“) und Unterbeschäftigung („,underemployment“) siehe ILO (2013). kommensländern die Abgrenzung zur Arbeitsmarktbeteiligung bei den Jugendlichen bis 14 Jahren und bei älteren Personen über 64 Jahren relativ eindeutig gelingt, ist diese Grenzziehung in vielen ärmeren Weltgegenden mitnichten einfach bzw. überhaupt möglich. Es gibt weit verbreitet Kinderarbeit (in Summe ca. 150 Mio. global gesehen; ILO 2017a) und aufgrund fehlender Altersversorgung gibt es auch viele Menschen, die im hohen Alter noch der einen oder anderen Arbeit nachgehen müssen. Über den Beobachtungszeitraum zeigt sich die markanteste Änderung an der Lohnarbeit, die um ca. 5 Prozentpunkte auf ca. 24 Prozent der Gesamtbevölkerung ansteigt; in absoluten Zahlen ein Zuwachs von ca. 760 Mio. LohnarbeiterInnen auf 1,78 Mrd. Dieser Zuwachs ist in allen Weltregionen zu verzeichnen, besonders ausgeprägt war er in den upper-middle income countries (hier stieg der Anteil der Lohnabhängigen von ca. 21 Prozent auf ca. 32 Prozent an). Am stärksten in den Arbeitsmarkt integriert sind LohnarbeiterInnen, wiewohl es auch hier enorme Unterschiede zwischen mehr oder minder unkündbaren, ganzjährig Vollzeiterwerbstätigen und prekär Beschäftigten (Stichwort: Teilzeit, Befristung, Praktika etc.) gibt. Neben den LohnarbeiterInnen weisen die ILO-Daten sogenannte vulnerable employed aus, das sind Soloselbstständige und mithelfende Familienangehörige (deren Anteil an der Gesamtbevölkerung sich beinahe halbiert hat). Diese Kategorie an Beschäftigten 
spielt v. a. in den Ländern mit mittleren und niedrigeren Nationaleinkommen eine erhebliche Rolle. Aus Sicht der Erwerbsintegration daran anschließend kommen Arbeitslose, die, global gesehen, mit ca. 190 Mio. eine eher untergeordnete Kategorie darstellen. ${ }^{9}$ Die Grenzen zur inaktiven Bevölkerung im Alter von 15-64 Jahren sind fließend, Stichworte dazu sind stille Reserve und unterbeschäftigte Teilzeitkräfte; diese beiden Gruppen machten in der EU 2016 ca. 20 Mio. Personen aus, neben weiteren 20 Mio. Arbeitslosen (Eurostat 2018). Wird die Entwicklung nach Frauen und Männern separat dargestellt, so zeigen sich folgende Unterschiede: Der Anteil der Lohnarbeiter (an der männlichen Bevölkerung) ist mit 28 Prozent um etwa 9 Prozentpunkte höher als bei den Frauen; auch der Anteil der Unternehmer mit abhängig Beschäftigten und der Soloselbstständigen ist bei den Männern deutlich höher als bei den Frauen; spiegelbildlich ist die Inaktivität der Männer (15-64 Jahre) mit 10,5 Prozent um etwa 18 Prozentpunkte niedriger als jene der Frauen.

In der nachfolgenden Abbildung 2 wird der Versuch unternommen, die begrifflichen Kategorien der vorangegangenen Abbildung noch weiter zu verfeinern, allerdings werden nur noch die subalternen ArbeiterInnen in den Blick genommen: Die Lohnarbeit wurde in einem ersten Schritt in Vollzeit-, Teilzeit- und befristete (inkl. Saison-, Projekt-, Gelegenheitsarbeit) Beschäftigung unterteilt. ${ }^{10}$ In einem weiteren Schritt wurde der Anteil der informell Beschäftigten herausgerechnet (Quelle v.a. ILO 2018a). ${ }^{11}$ Dieser Anteil ist - aufgrund von naheliegenden erheblichen Erfassungsproblemen - äußerst

9 Diese vergleichsweise niedrige Zahl spiegelt nicht etwa das geringe Ausmaß an globaler Arbeitslosigkeit wider, sondern vielmehr das niedrige Absicherungsniveau im Falle von Arbeitslosigkeit in vielen Weltgegenden.

10 Während es für Hocheinkommensländer gute Statistiken dazu gibt, die einen Anteil der prekären Beschäftigung (prekäre Teilzeit, Leiharbeit, befristet Beschäftigte) von 20-25 Prozent der Gesamtbeschäftigung ausweisen, fehlen diese detaillierten Statistiken in anderen Ländern. Daher wurde diese prekäre Lohnarbeit approximiert über (1) den Anteil der Teilzeitbeschäftigung (weniger als 35 Wochenstunden) an der Lohnarbeit, dieser Anteil reicht von 16-26 Prozent (siehe ILO 2016b); und (2) über den Anteil an befristeten Beschäftigungsverhältnissen, der zwischen 10-50 Prozent an der gesamten Lohnarbeit liegt.

11 Weil dafür auf internationaler Ebene keine Informationen vorliegen, konnte eine weitere Unterteilung der LohnarbeiterInnen je nach ihrer Stellung in der Hierarchie im Produktionsprozess (Management, Leitungsfunktionen, ExpertInnen, einfache ArbeiterInnen mit keiner/wenig Entscheidungsautonomie) nicht vorgenommen werden. schwer einzugrenzen, insbesondere auf globaler Ebene (siehe weiterführend unten). ${ }^{12}$ Es wurde für die vorliegende Arbeit ein Ausmaß an informeller Beschäftigung von ca. 18 Prozent (Hocheinkommensländer) bis 85 Prozent (Niedrigeinkommensländer) angenommen, wobei sich die Prozentzahlen auf die Basis von Beschäftigung (d.h. Lohnarbeit, Kapital, Soloselbstständigkeit, mithelfende Familienangehörige) beziehen ${ }^{13}$ (ILO 2018a). Die weiteren Schritte waren die folgenden:

- Neben den Arbeitslosen gibt es Phänomene der Unterbeschäftigung und der stillen Reserve (discouraged workers, time-related underemployment, labour underutilization), Gruppen, die sich v.a. aus den Inaktiven (15-64 Jahre) speisen im Sinne einer stillen Reserve. ${ }^{14}$

- Die Kinderarbeit (ca. 150 Mio.) wurde von der Bevölkerung zwischen o-14 Jahren abgezogen.

- Zudem werden global etwa 40 Mio. SklavenarbeiterInnen (forced labour und forced marriage) ausgewiesen (ILO 2017b), die ebenfalls berücksichtigt wurden.

- Zu guter Letzt kann noch ein kleiner Teil (ca. 3-5 Prozent der Gesamtbeschäftigung) im Alter über 65 Jahren ein Einkommen aus Lohnarbeit beziehen. ${ }^{15}$

Eine besondere Schwierigkeit besteht darin, dass viele Statuspositionen, v. a. in ärmeren Weltgegenden,

12 Die Schätzungen des informellen Sektors gehen in vielen Ländern sehr weit auseinander. Ein besonderes Beispiel ist Indien, ein Land, für das Werte zwischen 20,7 Prozent und 94 Prozent zu finden sind, bei China liegen diese zwischen 12-30 Prozent (OECD 2010). Zur Definition des informellen Sektors bzw. der informell Beschäftigten siehe ILO 2018a: 3ff. und weiterführend unten.

13 Für die ILO kommt das Gros an informeller Beschäftigung aus der Lohnarbeit, den Selbstständigen und Soloselbstständigen und den mithelfenden Familienangehörigen (hier zu 100 Prozent). Darüber hinaus wurde für die vorliegende Arbeit angenommen, dass auch ein gewisser Teil der informellen Beschäftigung (zwischen ca. 5-15 Prozent) aus der Inaktivität (15-64 Jahren) herrührt, ein Bevölkerungssegment, das v.a. in den ärmeren Weltgegenden sehr groß ist.

14 Angenommen wurde eine Größe von ca. 20 Prozent der Inaktiven (15-64 Jahre) bzw. 100-200 Prozent der Arbeitslosen; in der EU standen 2016 etwa 20 Mio. Arbeitslosen ca. 20 Mio. Unterbeschäftigte gegenüber (ILO (2008: 31); die Schätzungen schwanken sehr stark, zwischen 50 Prozent der Arbeitslosen in Panama und 500 Prozent in Tansania (EUROSTAT 2018), ILOSTAT, Time-related underemployment.

15 Durch den Schritt von Abbildung 1 zu Abbildung 2 wurde die Grundgesamtheit (das ist einmal die Gesamtbevölkerung von ca. 7,53 Mrd. Personen, das andere Mal ein „erweitertes Arbeitsangebot" bzw. die Gruppe der subalternen Arbeiter von ca. 4,23 Mrd. Personen) um ca. 3,3 Mrd. Personen verkleinert. 
Abbildung 2: Subalterne Arbeiter - Lohnarbeit, prekäre Beschäftigungsformen, Überschussbevölkerung (2017, nach BNE-Gruppen, in Mio. Pers., globale Summe: 4,23 Mrd.)

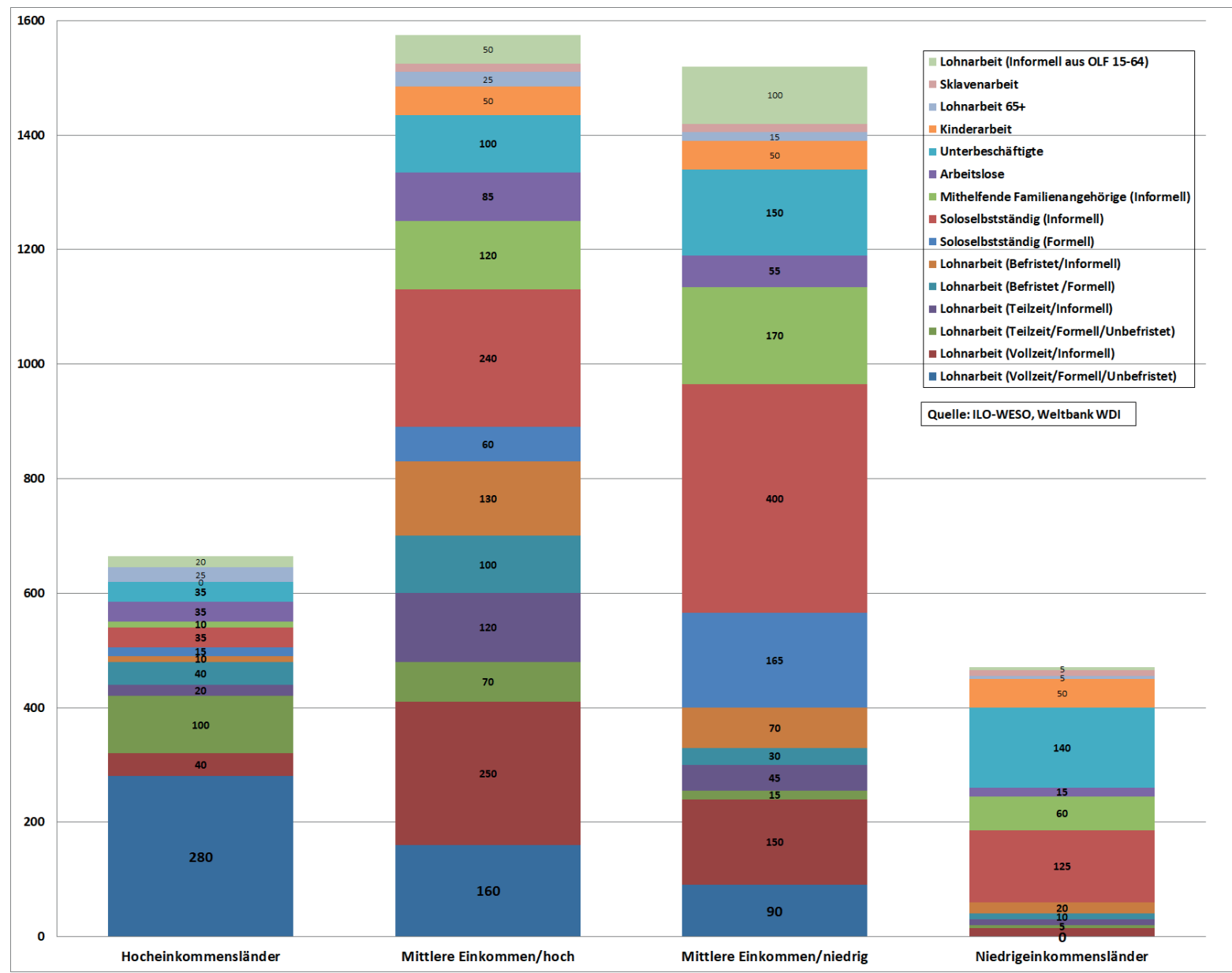

aber natürlich nicht nur dort, nicht genau zu trennen sind, weil sie als Mischformen vorkommen: jemand verbindet etwa einen kleinen Teil seines Einkommens im formellen Sektor mit Schwarzarbeit; einen anderen Teil des Jahres arbeitet er oder sie etwa in der Subsistenzwirtschaft. ${ }^{16}$

Was lässt sich nun aus dieser Aufschlüsselung - bei der es, und dies sei hier besonders betont, v. a. um die Größenordnungen und nicht um die Details gehen sollte - für die Zusammensetzung der globalen subalternen

16 Die ILO „löst“ das Problem bei ihren Darstellungen der Beschäftigung mit einer Hierarchisierung, d. h. es wird bei multiplen Beschäftigungsverhältnissen ein main job (longest hours usually worked) als die dominante Position angenommen. Darüber hinaus wird bei der Beschäftigung ein sehr niedriger Schwellenwert angesetzt, nämlich mindestens 1 Stunde Beschäftigung in einer kurzen Referenzperiode. Dies führt dazu, dass auch zeitlich sehr kurze Beschäftigungsverhältnisse in den Blick treten, andererseits wird (wenn nicht mit Arbeitszeitgewichten gearbeitet wird) die Beschäftigungsstatistik mit dieser „Kopf-Zählung“ künstlich „aufgebläht“(siehe ILO 2013).
ArbeiterInnen (Lohnarbeit, Prekariat, Überschussbevölkerung) schließen?

- Von allen subalternen ArbeiterInnen (4,23 Mrd.) arbeiten ca. 3,1 Mrd. in den Ländern mit mittleren Einkommen, das sind ca. 73 Prozent; d. h. in einer globalen Perspektive fällt sowohl in den reichen als auch in den sehr armen Länder nur der geringere Teil der globalen, subalternen Arbeit an.

- Global gesehen ist die Gruppe der LohnarbeiterInnen, die Vollzeit und unbefristet im formellen Sektor arbeitet (mit insgesamt 530 Mio. Personen, das sind 12,5 Prozent von allen subalternen Arbeitern), eine vergleichsweise kleine Gruppe. Relevant ist diese Gruppe darüber hinaus nur in den Hocheinkommensländern (dort umfasst sie etwa 42 Prozent aller Subalternen), in allen anderen Weltgegenden liegt ihr Anteil bei unter 10,2 bzw. 5,9 Prozent (Länder mit mittleren Einkommensniveaus, hoch und niedrig).

- Die viel größere Gruppe ist jedoch jene der prekär und informell Beschäftigten: 880 Mio. informell 
beschäftigte LohnarbeiterInnen, 800 Mio. informell beschäftigte Soloselbstständige, 190 Mio. formelle Teilzeit- und 180 Mio. formelle befristet Beschäftigte, 360 Mio. informell beschäftigte mithelfende Familienangehörige, 425 Mio. Unterbeschäftigte (stille Reserve), 240 Mio. Soloselbstständige im formellen Sektor, 190 Mio. Arbeitslose, 175 Mio. informell Beschäftigte im Bereich der offiziell als inaktiv Ausgewiesenen (15-64 Jahre), 150 Mio. KinderarbeiterInnen und 40 Mio. SklavenarbeiterInnen.

Daraus folgt: Ca. 10-15 Prozent der Subalternen sind über ein formelles Arbeitsverhältnis in den Arbeitsmarkt integriert, die anderen knapp 85-90 Prozent sind prekär und informell in der einen oder anderen Form. ${ }^{17}$ Das ist die zentrale Erkenntnis in diesem Abschnitt und auch die empirische Begründung für G. Standing, nicht vom globalen Proletariat, sondern vom globalen Prekariat zu sprechen. ${ }^{18}$ Marcel van der Linden kommt zu einem recht ähnlichen Ergebnis: Auf die Frage, ob die Prekarisierung ein globales Phänomen sei, antwortet er in einem Interview mit dem Neuen Deutschland:

„Das Normalarbeitsverhältnis, wie wir es einige Jahrzehnte gekannt haben, war eine temporäre Erscheinung für einen kleinen Teil der Welt. Das hat es 50 Jahre für 20 Prozent der Weltbevölkerung gegeben, jetzt wird es abgebaut. Damit kehrt der Zustand zurück, der vorher herrschte. Die Norm im Weltkapitalismus ist die Prekarität. Die meisten Menschen in der Welt haben immer, wenn sie Lohnarbeit verrichtet haben, unter prekären Verhältnissen gearbeitet und tun es auch jetzt noch. Feste Arbeitsverträge gibt es zum Beispiel in Indien nur bei 3 bis 4 Prozent der Arbeiterschaft. Was wir Normalarbeitsverhältnis nennen, ist eigentlich atypisch. Und was wir atypische Beschäftigung nennen, ist die Normalität." (Van der Linden 2017)

17 Dies entspricht nicht den Darstellungen der ILO, siehe Srnicek/Williams 2015, Kapitel 5, FN 37 und 43, S. 331f; auch Foster et.al. $(2011,2016)$ kommen in ihrer Analyse von Lohnarbeit und globaler Reservearmee (d.h. bei ihnen: vulnerable Beschäftigung, Arbeitslose, Inaktive 25-54) zu einem anderen Ergebnis, es standen 2015 etwa 1,66 Mrd. Lohnarbeitern (ohne prekär Beschäftigte) ca. 2,3 Mrd. Personen der Reservearmee gegenüber. Die Unterschiede ergeben sich durch die hier angestellte stärkere Aufgliederung von Lohnarbeit in Teil-/Vollzeit- und formell/ informell Beschäftigte.

18 Bei G. Standing finden sich zwar viele einzelne Hinweise auf nationale Verbreitung verschiedener Formen des Prekariats, aber er nennt keine globalen Daten. In dieser Form finden sich diese Zahlen zum ersten Mal.

\section{b) Informelle Beschäftigung}

Wie in der nachfolgenden Abbildung 3 (ILO 2018a: 13) $\mathrm{zu}$ sehen ist, variiert das Ausmaß an informeller Beschäftigung als Anteil an der Gesamtbeschäftigung (d. h. Lohnarbeit, Unternehmer, Soloselbstständige, mithelfende Familienangehörige) global gesehen zwischen ca. 20 und über 90 Prozent. Insgesamt gibt es weltweit ca. 2 Mrd. informell Beschäftigte, das sind 61,2 Prozent der Gesamtbeschäftigung (ILO 2018a). Naturgemäß sind allerdings die Größe des informellen Sektors bzw. das Ausmaß an informeller Beschäftigung schwer abzugrenzen bzw. zu ermitteln. Die Definition des informellen Sektors geht dabei von einem Unternehmen bzw. den Charakteristika eines bestimmten Arbeitsplatzes aus, jene der informellen Beschäftigung von einem bestimmten Beschäftigungsverhältnis. ${ }^{19}$

Der Anteil an informell beschäftigten Frauen ist etwas kleiner als jener der Männer. Die Integration in den formalen Arbeitsbereich ist im Haupterwerbsalter am größten, sie sinkt an beiden Rändern der Altersverteilung. In der Abbildung 3 (rechte Darstellung: ILO 2018a: 22) ist die Verteilung der informell beschäftigten Männer und Frauen auf die Positionen Lohnarbeit, Kapital, Soloselbstständigkeit und mithelfende Familienmitglieder zu sehen. Dabei fällt auf, dass der Großteil der informellen Beschäftigung bei den Soloselbstständigen anfällt. Sektoral gesehen ist der größte Teil der informellen Beschäftigung im primären Sektor (Landwirtschaft) zu finden, dies gilt auch für

19 Folgende Kriterien für den informellen Sektor werden dabei genannt: Es handelt sich um ein Unternehmen, einen Arbeitsplatz, das/der nicht den privaten Haushalten oder dem Staat angehört; es liegt keine separate legale Einheit unabhängig vom Eigentümer vor; die Produktion erfolgt nicht überwiegend für den Eigenbedarf; es gibt keine Registrierung bei den Steuer- und Sozialversicherungsbehörden; es liegt keinerlei formelle Buchhaltung vor (wenn zu den beiden letzten Kriterien keine Informationen vorliegen, dann wird folgende Regel angewandt: Wenn weniger als sechs Beschäftigte und keine fixen Betriebsgebäude vorliegen, dann handelt es sich um ein Unternehmen des informellen Sektors). Die informelle Beschäftigung wird folgendermaßen definiert: Bei mithelfenden Familienangehörigen wird angenommen, dass sie alle informell beschäftigt sind; bei Arbeitgebern, Soloselbstständigen und Mitgliedern einer Produktionsgenossenschaft liegt dann informelle Beschäftigung vor, wenn die ökonomische Einheit Teil des informellen Sektors ist; bei Lohnarbeitern schließlich wird informelle Beschäftigung angenommen, wenn keine Sozialversicherungsbeiträge bezahlt werden bzw. keine Ansprüche auf bezahlten Urlaub bzw. Krankenstand bestehen (siehe ILO 2018). 
Abbildung 3: Anteile an informeller Beschäftigung (in \% der Gesamtbeschäftigung)
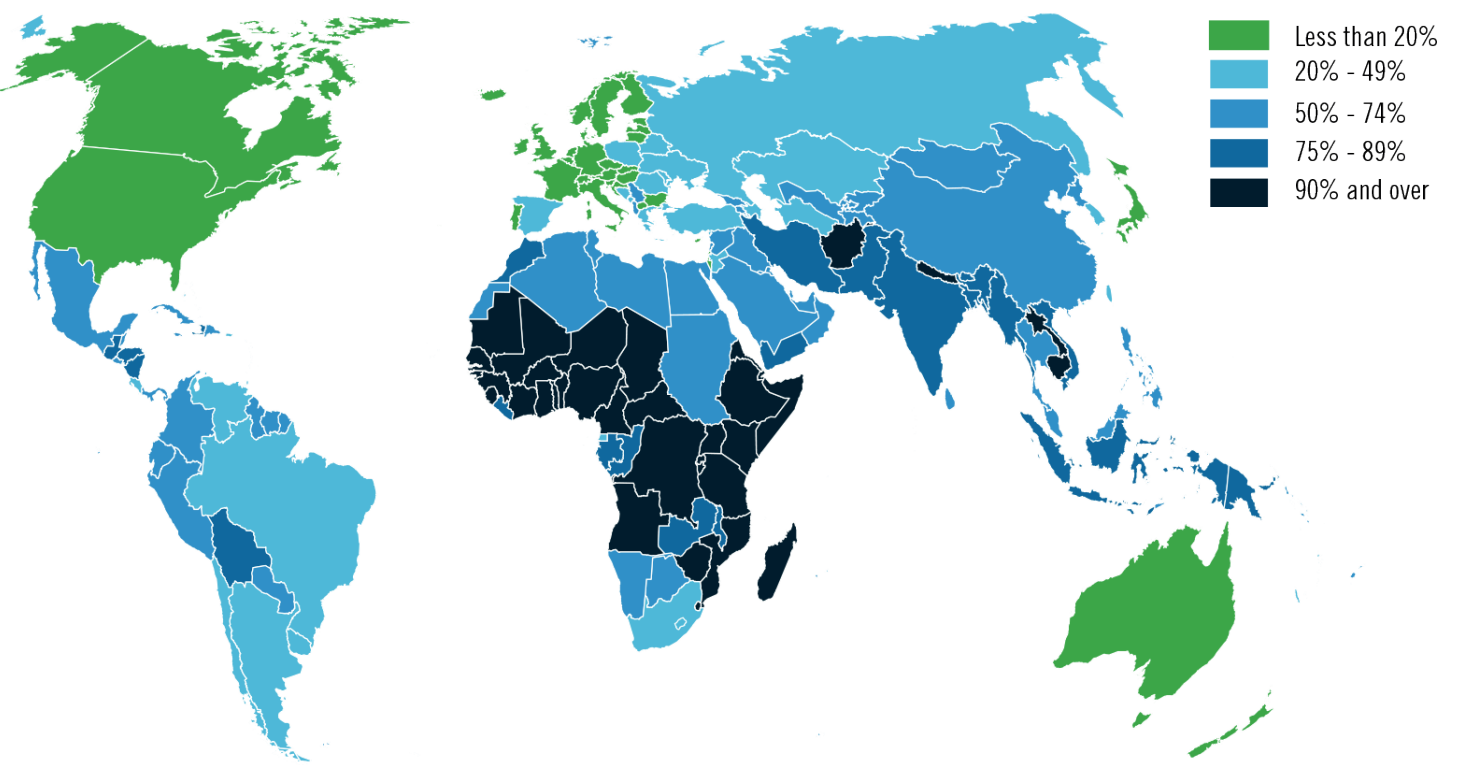

Quelle: ILO 2018a: 13

den Anteil an Soloselbstständigen und mithelfenden Familienmitgliedern. Nur in Niedrigeinkommensländern und lower-middle income countries ist der Anteil an informeller Beschäftigung in allen Sektoren relativ hoch (ILO 2018b: 34).

Eine genauere Beschreibung der Rolle der informellen Beschäftigung in einer ökonomischen und politischen Analyse der Entwicklung von Weltregionen könnte auf nationaler oder subnationaler bzw. regionaler Ebene erfolgen. So weist beispielsweise Cane (2017) in einer Analyse der Informal economy and India's working class darauf hin, dass die Politik der staatskapitalistischen Entwicklung unter J. Nehru nur eine kleine indische Arbeiterklasse entstehen ließ, der Großteil der arbeitenden Bevölkerung jedoch in informellen Beschäftigungsformen ${ }^{20}$ gefangen blieb, die auch einen ständigen Wechsel von landwirtschaftlicher Produktion und städtischen Gelegenheitsarbeiten, von Beschäftigung im formellen und informellen Bereich, mit sich brachte. Mit den neoliberalen Reformen der 1980er- und 9oer-Jahre wurden allerdings die entstandenen Enklaven der formalen Beschäftigung durch Angriffe der Unternehmen auf Rechte der ArbeiterInnen, Deindustrialisierung, die umfassende Anwendung von contract labour (Arbeiter werden über Verleihfirmen vermittelt), aber auch durch die Errichtung von

20 Eine genauere Analyse der Non-standard forms of employment in India ist zu finden in Srivastava 2016. Siehe auch Chen 2012.
Sonderwirtschaftszonen weitreichend ausgehöhlt (zu anderen Beispielen auf Länderebene: Burchardt et. al. 2013; Webster et. al. 2008).

\section{c) Soziale Absicherung}

In einer umfassenden aktuellen Darstellung der Systeme der sozialen Sicherheit geht die ILO (2017c) der Frage nach, wie sich die Schutzniveaus global entwickelt haben und wo wir in Bezug auf die UN-Ziele zur nachhaltigen Entwicklung stehen. Bereits ein Blick auf die Ausgaben für soziale Sicherheit (ohne Gesundheit, siehe Abbildung 4 unten) zeigt erhebliche Unterschiede nach Weltregionen - konkret schwanken diese zwischen weniger als 5 Prozent des BIP bis über 15 Prozent. ${ }^{21}$ Diese Unterschiede zeigen sich auch bei der Frage, wie viele Personen in der Bevölkerung überhaupt von einem Transfer der sozialen Sicherheit erfasst sind: In Europa und Zentralasien sind dies 84,1 Prozent, in den Ländern der Sub-Sahara jedoch nur 12,9 Prozent.

21 Von wenigen Ausnahmen abgesehen zeigt sich in der längerfristigen Entwicklung ein Anstieg der Ausgaben für soziale Sicherheit (in \% des BIP) in beinahe allen Ländern der Welt; allerdings sind die Ausgabenzuwächse sehr unterschiedlich, wie es auch die Ausgangsniveaus sind. Beispielsweise stiegen die Ausgaben in Mexiko im Zeitraum 1985-2011 von 1,7 Prozent auf 7,7 Prozent des BIP, in Griechenland zwischen 1980 und 2016 von 9,9 Prozent auf 27 Prozent (OECD, ILO Social Security Database). 
Abbildung 4: Ausgaben für soziale Sicherheit - global

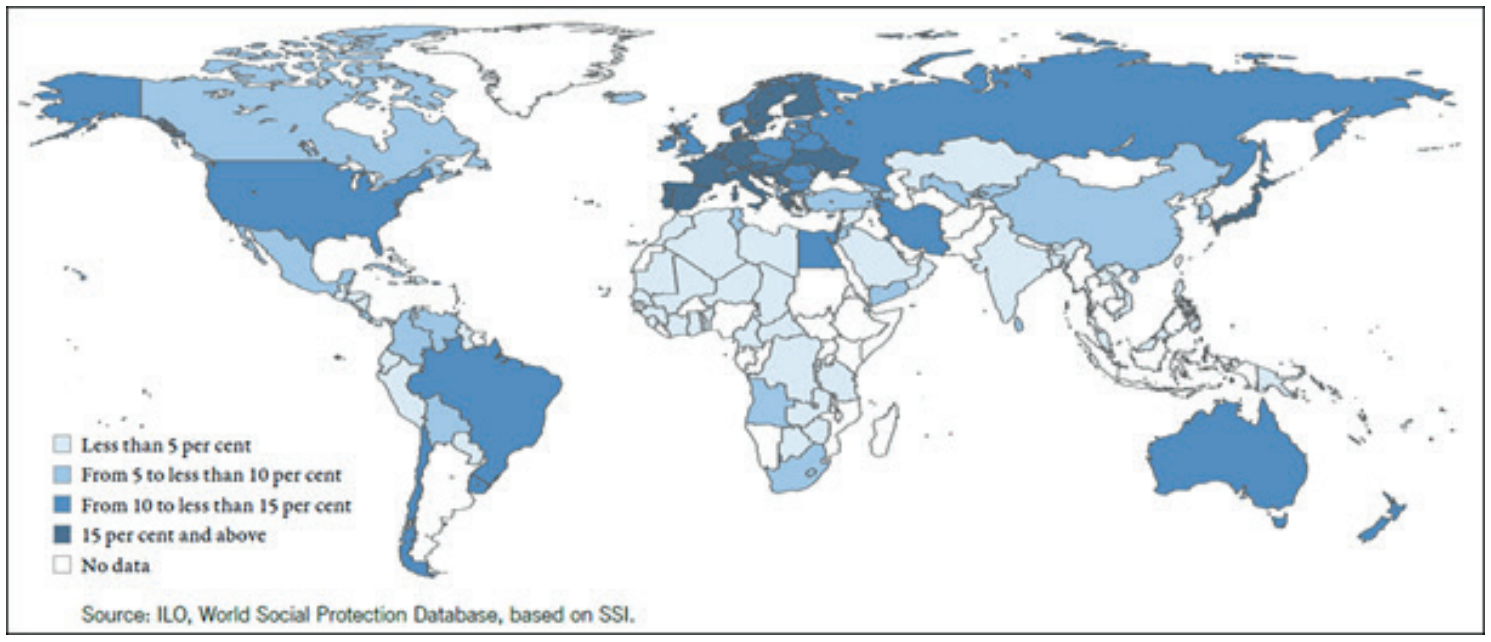

Quelle: ILO $2017 \mathrm{C}$

Abbildung 5: Nationale Einkommensverteilungen im globalen Vergleich
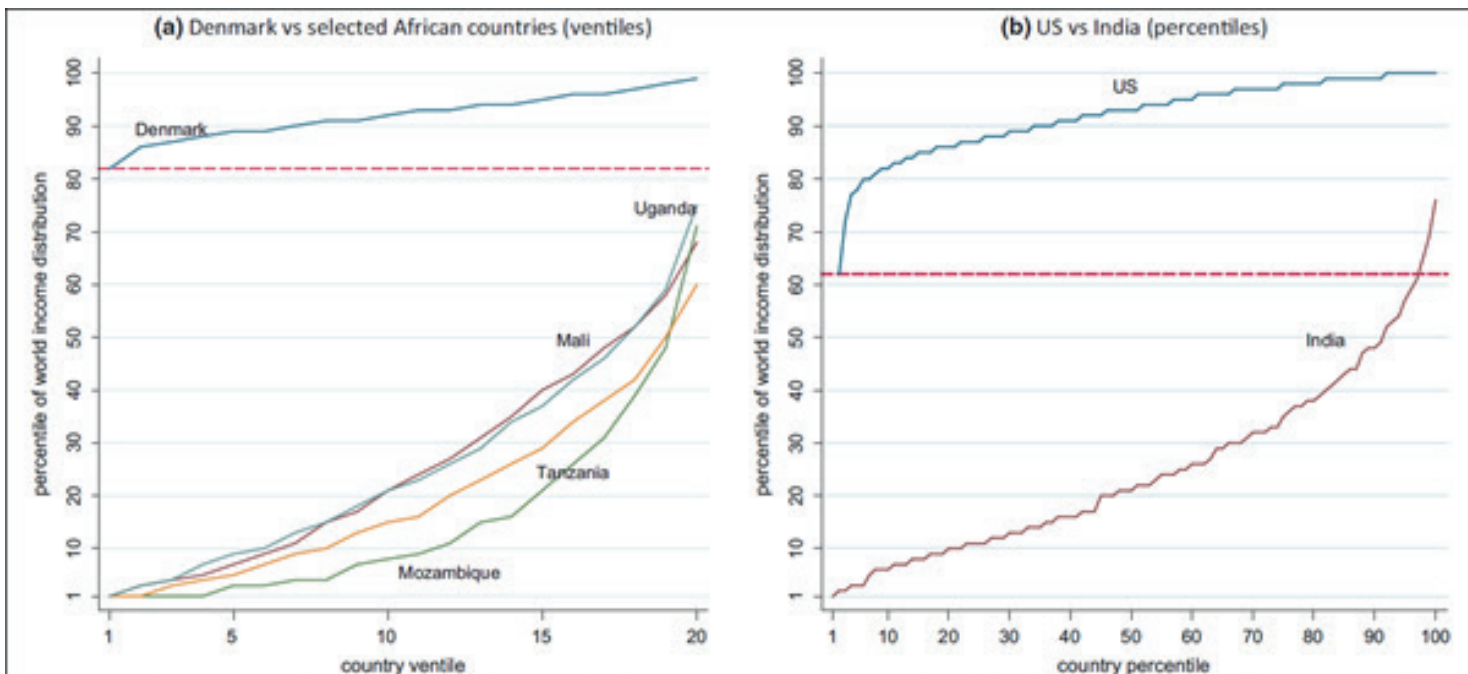

Source: Own calculations using World Income Distribution (WYD) database. Note: Based on national household surveys; adjusted for price differences between the countries using most recent PPPs. Year 2005.

Quelle: Milanovic 2012: 128

d) Globale Einkommensverteilung - Beschäftigung nach economic classes (ILO)

Wie stellt sich die Verteilung der Einkommen auf einer globalen Skala dar und was ließe sich daraus für die Organisierungsfähigkeit der subalternen Klasse schließen? Die Armen in den reichen Ländern verfügen etwa über ein ähnliches kaufkraftbereinigtes Einkommen wie die Reichen in den sehr armen Ländern, und die Armen in den armen Ländern sind sehr viel ärmer als die Armen in den reichen Ländern. Dieses Bild wird in den genaueren Analysen von Lakner/ Milanovic (2015) bestätigt (siehe Abbildung 5, Milanovic 2012: 128): Vergleicht man etwa das durchschnittli- che Einkommen im untersten Ventil (20 Prozent der Verteilung) von Dänemark mit jenem in Tansania, Uganda, Mali oder Mosambik, dann zeigt sich: Die sehr Armen in Dänemark, die etwa am 82. Perzentil der globalen Einkommensverteilung liegen (82 Prozent der Weltbevölkerung sind also ärmer als diese Gruppe), sind ca. achtzigmal reicher als die sehr Armen in den genannten afrikanischen Staaten. Vergleicht man die untersten Perzentile (1 Prozent) zwischen den USA und Indien, so sind die Unterschiede etwa 1:60. Andererseits verfügt das 95. Perzentil in Indien etwa über ein Einkommen, das vergleichbar ist mit dem untersten Perzentil in den USA. Insgesamt scheinen die Abstände 
am unteren Ende der Verteilung zwischen einzelnen Ländern deutlich größer zu sein als oben.

Die ILO (ILO 2016) weist seit einigen Jahren die Entwicklung der Beschäftigungsanteile nach economic classes, also nach Einkommenshöhen, aus. Dabei zeigt sich, dass der Anteil an Personen, die in extremer Armut, ${ }^{22}$ also in einem Haushalt leben, bei dem das Pro-Kopf-Haushaltseinkommen unter USD 1,9 täglich liegt, sowohl in den Ländern mit niedrigen als auch mit mittleren Einkommen seit 1992 deutlich zurückgegangen ist; die Anteile der anderen Einkommenskategorien steigen demgegenüber kontinuierlich an (ILO 2016: 18). Die extrem Armen arbeiten dabei häufig als Soloselbstständige und in der Landwirtschaft, jene Personen mit einem Einkommen über USD 13 beziehen ihre Einkommen häufig durch Lohnarbeit und im Dienstleistungsbereich (Kapsos/Bourmpoula 2013: 7).

Betrachtet man allerdings nur die mittleren Einkommensbereiche (um den Median herum) in den verschiedenen Ländern, dann zeigt sich, dass die Abstände deutlich geringer werden. Dies gilt insbesondere, wenn etwa die Realeinkommen von qualifizierten Facharbeitern betrachtet werden. In diesem Fall reduzieren sich die Unterschiede, etwa zwischen London und Neu-Delhi auf 1:3,3 bzw. 5,8 (Milanovic 2012: 127, 130). Daraus kann gefolgert werden: Auch wenn die Einkommen der unteren Schichten subalterner Arbeit im Vergleich zwischen den verschiedenen Weltregionen noch immer extrem ungleich verteilt sind, näherten sich die Einkommen um den Median und korrigiert um Qualifikationsniveaus doch in den vergangenen Jahrzehnten deutlich an. Das heißt, die Abstände werden global gesehen kleiner und damit vielleicht auch - so die implizite Hoffnung - die Unterschiede in den Klasseninteressen.

\section{Formierung/Organisierung subaltern Arbeitender}

Die Formierung der ArbeiterInnenklasse wurde sowohl von politischen AkteurInnen als auch von sozialwissenschaftlichen BeobachterInnen in ihren hegemonialen Varianten vor allem mit einem wie auch immer genau

22 Die von der Weltbank vorgegebenen Armutsschwellenwerte sind als „inhumanely low" $\mathrm{zu}$ bewerten, wahrscheinlich sollten eher Werte von USD 5 oder USD 10 angenommen werden (Selwyn 2014). Es hat sich in empirischen Studien zu Lateinamerika gezeigt, dass erst bei Einkommenshöhen von USD 10 und darüber ein erneutes Abgleiten in die Armut dauerhaft verhindert werden kann (Fischer 2017: 43). bestimmten industriellen Kern und den entsprechenden Gruppen von ArbeiterInnen, Organisations- und Aktionsformen verbunden. Andere Gruppen wurden von Gewerkschaften und politischen Parteien der Linken entweder advokatorisch „serviciert“ oder als politisch unsichere KantonistInnen oder mobilisierbare Manövriermasse der GegnerInnen betrachtet. Das „Making of“ der ArbeiterInnenklasse (Thompson 1966) ging daher sowohl ideologisch als auch organisatorisch mit einer Vereinheitlichung, Disziplinierung und entsprechenden Exklusionsmechanismen einher, die vielköpfige Hydra (Linebaugh/Rediker 2008) wurde so zu einer homogenen Gruppe respektabler ArbeiterInnen imaginiert (vgl. etwa Bescherer 2013, Eiden-Offe 2017). Mit der Prekaritätsdiskussion tauchen entsprechende Motive und Argumentationsmuster wieder auf. Die Diskussion fokussiert dabei vor allem auf zwei Fragen: 1) Sind Prekäre überhaupt organisierbar bzw. wie (un-) wahrscheinlich ist eine Selbstorganisation von Personen in derart instabilen Lebenslagen? 2) Handelt es sich beim „Prekariat" um eine neue „Klasse“ (Standing) mit ganz eigenen Interessen und sind daher Aktionsformen zu erwarten, die sich nicht nur unterschiedlich, sondern sogar in Abgrenzung zu einem als saturiert gezeichneten „Salariat“ (Standing) als einer Art „Arbeiteraristokratie" begreifen lassen?

Wir wollen uns im folgenden Abschnitt mit diesen beiden Fragen beschäftigen, im Anschluss auf Organisierungsprozesse von informell Erwerbstätigen eingehen und abschließend Schlussfolgerungen formulieren, die sich daraus aus unserer Sicht sowohl in sozialwissenschaftlicher als auch in politischer Hinsicht ergeben.

\section{a) „Unmögliches", „unwahrscheinliches" oder „verrücktes” Prekariat?}

Mit Bezug auf die Frage der Organisierbarkeit kann man idealtypisch die Positionen von Autoren wie Bourdieu, Castel oder Wacquant anderen AutorInnen wie Artus oder Candeias gegenüberstellen. Erstere könnte man als soziologische Pessimisten (sie würden sich wohl eher als Realisten sehen) bezeichnen, die darauf verweisen, dass ein Regime der Angst und der permanenten Unsicherheit eher Apathie, Rückzug und individuelle Überlebensstrategien als widerständiges Handeln fördert und Statuspositionen wie Arbeitslosigkeit oder eine verweigerte Arbeitsoder Aufenthaltserlaubnis nicht mit einer positiven Selbstidentifikation und kollektivem Handeln, sondern mit der Hoffnung einer baldigen (individuellen) 
Überwindung dieses Status einhergehen. Bourdieu hat in diesem Zusammenhang etwa die Entstehung der Arbeitslosenbewegung in Frankreich als "gesellschaftliches Wunder" bezeichnet. Candeias verweist dagegen darauf, dass permanente Unsicherheit und vielfältige Segmentierungen bzw. Spaltungslinien zum „Normalzustand“ der ArbeiterInnenklasse gehören und auch auf die Formierungsphase der klassischen ArbeiterInnenbewegung in Westeuropa zutreffen. Außerdem fanden zum Beispiel in Deutschland viele der innovativeren Arbeitskämpfe der vergangenen Jahre in weiblich und migrantisch geprägten Bereichen prekärer Beschäftigung statt, die lange als unorganisierbar galten. Artus (2015) spricht in diesem Zusammenhang auch von „verrückten“, weil scheinbar aussichtslosen Kämpfen. Einem Blick auf ein „Prekariat", das von Anomie und Apathie geprägt ist und als einzige mögliche Aktionsform mangels Machtressourcen wiederkehrende eruptive Gewalt sieht, die sich gegen Symbole der Konsumgesellschaft (Sachbeschädigungen, Plünderungen) oder des Staates (z. B. aufgrund wiederholter Polzeigewalt) richtet, setzt Candeias das Bestreben entgegen, nach verallgemeinerbaren Erfahrungen und Motiven für kollektives Handeln zu suchen, für das er verschiedene Fallbeispiele anführt (Candeias 2007, Candeias/ Steckner 2015).

Längerfristige Organisierung und erfolgreiche Durchsetzung von Interessen prekär oder informell Beschäftigter ist voraussetzungsvoll und unwahrscheinlich, findet aber trotzdem in verschiedenen Formen statt. In der Forschung wird in diesem Zusammenhang vielfach auf das Konzept der Machtressourcen zurückgegriffen, das vor allem Beverly Silver in Anlehnung an Erik Olin Wright entwickelt hat, im deutschen Sprachraum wäre die Forschungsgruppe in Jena zu nennen, die den Machtressourcenansatz seit einigen Jahren verwendet und weiterentwickelt (Silver 2005; Britwum 2018). Hier werden verschiedene Formen von Machtressourcen bzw. -quellen unterschieden, die mit Positionen in der Ökonomie sowie politischen Gelegenheitsstrukturen typischerweise einhergehen. ${ }^{23}$

23 Der Machtressourcenansatz wurde 2018 in mehreren Ausgaben des Global Labour Journals kontroversiell diskutiert. Auf diese Debatte bzw. die Argumente zu Stärken und Schwächen bzw. Grenzen des Ansatzes können wir im Rahmen dieses Artikels nicht eingehen. Wir verweisen an dieser Stelle auf die Replik von Schmalz/Ludwig/Webster (2019), die auf die kritischen Einwände eingeht.
So können verschiedene Formen von struktureller Macht Gelegenheiten eröffnen. Wright unterscheidet hier etwa market bargaining power aufgrund spezifischer Skills und workplace bargaining power im Zusammenhang mit strategisch wichtigen Positionen in Produktionsprozessen bzw. Güterketten, Silver spricht auch von logistical power, die nicht an den Arbeitsplatz gebunden ist und sich auf die Möglichkeit bezieht, z. B. Transportwege oder Kommunikationskanäle zu blockieren. Organisatorische Möglichkeiten (associational power) bezeichnen eine weitere Dimension, hier geht es vor allem um finanzielle und personelle Kapazitäten, um schlagkräftige Organisationen, Koalitionen oder Netzwerke aufzubauen und aufrechtzuerhalten. Institutionelle Macht meint Zugang $\mathrm{zu}$ politischen Prozessen, Eingebundenheit in das politische System, z. B. über Tarifverhandlungen, parlamentarische Vertretung oder enge Verbindungen zu politischen Parteien. Gesellschaftliche Macht bezieht sich auf die Möglichkeit und Fähigkeit, sich auf verschiedene breit geteilte Normen und die entsprechenden communities zu beziehen und damit auch Gehör zu finden. Chun spricht in diesem Zusammenhang von symbolischer Macht, „symbolic leverage“ bezeichnet ein Framing, das z. B. Fragen der Ausbeutung auf dem Arbeitsplatz mit breiteren sozialen Themen und moralischen Rechtfertigungen verknüpft und so Diskurse generiert, die breite gesellschaftliche Debatten um bürgerliche, politische und soziale Rechte ermöglichen, in die das Ausgangsthema eingebettet wird (Chun 2009). Vor allem symbolische und logistische Machtressourcen können von Gruppen erfolgreich eingesetzt werden, die über keine oder wenig strukturelle und organisatorische Macht verfügen, was wohl für große Teile prekär und informell Beschäftigter zutrifft. Alle Machtquellen und -ressourcen stellen allerdings nur Möglichkeiten dar, die auch ergriffen werden müssen. Die erfolgreiche Kombination verschiedener Machtressourcen in einem konkreten politischen und sozialen Raum hängt daher nicht zuletzt von strategischen Kapazitäten von kollektiven Akteuren ab (Webster 2015).

Verschiedene Formen und Dimensionen von Prekarität bieten unterschiedliche Möglichkeiten für kollektives Handeln, das sich an Anlässen entzünden kann, die in unterschiedlichen Lebensbereichen $\mathrm{zu}$ verorten sind, denn „prekär meint nicht nur Arbeits- und Lebensverhältnisse ohne existenzsicherndes Einkommen. Es geht auch um mangelnde Anerkennung der Arbeit und der Person, um betriebliche und soziale Isolierung, mangelnden Sozialversicherungsschutz und fehlende Qualifizie- 
rungsmöglichkeiten, um erschwerten Zugang zu oder die Verteuerung von öffentlichen Dienstleistungen und Sozialer Infrastruktur, um Verdrängung durch explodierende Mieten etc." (Candeias/Steckner 2015: 34)

Es ist daher davon auszugehen, dass es nicht die eine und einzige Form politischer Artikulation von Prekären gibt (z. B. Riots, bei denen der Sachschaden eine Art Verhandlungsmasse darstellt oder Blockaden bzw. soziale Streiks), sondern eine Vielfalt mehr oder weniger sichtbaren, individuellen und kollektiven Alltagshandelns. Aktionsformen von Personen in prekären Lebenslagen reichen von einzelnen widerständigen Akten gegenüber Behörden oder Unternehmen über verschiedene polyanische Proteste gegen (Re-)Kommodifizierung (wie z. B. gegen die Privatisierung der Wasserversorgung in Cochabamba, Bolivien), verschiedene Formen von Riots (z. B. Food Riots aufgrund der Einschränkung oder Beendigung von Lebensmittelsubventionen oder IMF Riots gegen Strukturanpassungsprogramme in Lateinamerika in den 1980er-Jahren) bis hin zu Marx'schen Arbeitskämpfen, die sich an Ausbeutungsfragen orientieren (vgl. etwa Webster et. al. 2008; Silver 2005; Lindell 2010).

Eine umfassende Auseinandersetzung mit verschiedenen Gründen für und Formen von kolletivem Handeln von informell und prekär Beschäftigten ist in diesem Artikel nicht möglich (vgl. etwa Schurman/Eaton 2012 und Eaton/Schurman/Chen 2017 mit Beispielen aus verschiedenen Ländern und Sektoren). Wir beschränken uns daher auf die (Selbst-)Organisation von prekär Erwerbstätigen und ihre organisierte Interessenvertretung. Wir teilen die Ausgangsposition der soziologischen Realisten, dass eine Reihe von Barrieren kollektives Handeln von Prekären erschwert, das gilt aber auch zunehmend für formell Beschäftigte. Der gewerkschaftliche Organisationsgrad (inklusive informell Beschäftigte) beträgt weltweit nur rund 7 Prozent (Van der Linden 2016: 203). Neben gewerkschaftlicher Organisierung und klassischen Arbeitskämpfen spielen im Zusammenhang mit der Artikulation und Bündelung der Interessen informell Beschäftigter auch andere Formen der Organisierung eine Rolle. Gewerkschaftliche Organisierung über den konkreten Arbeitsplatz ist nur eine spezielle Form neben anderen, die über andere Orte, wie z. B. das Wohnviertel und verschiedene (Teil-)Identitäten, erfolgen. Neben der gewerkschaftlichen Durchsetzung von Interessen spielen verschiedene Formen von Kooperativen, Versicherungen oder Plattformen, die den Zugang zu Krediten oder Weiterbildung ermöglichen, eine Rolle (Schurman/Eaton 2012). In einer Gesamtbetrachtung möglicher Reaktionsformen von Individuen und Haushalten auf Prekarität und Prekarisierung ist außerdem zu beachten, dass diese von der Größe und Zusammensetzung der Haushalte sowie von der Abhängigkeit von spezifischen Lohneinkommen und alternativen Einkommensquellen und Optionen auf Haushaltsebene abhängt und proaktives kollektives Handeln neben verschiedenen individuellen Reaktionsweisen (Rückzug bzw. Apathie, mehr Arbeit bzw. zweiter oder dritter Job, Migration u. a.) nur eine mögliche, voraussetzungsvolle Option darstellt.

Schließlich wäre bei aller Unwahrscheinlichkeit der (Selbst-)Organisierung von informell Beschäftigten auf eine strukturelle Verbesserung der Kommunikationsmöglichkeiten hinzuweisen: Für Marx waren die Parzellbauern ein Beispiel für eine zersplitterte Arbeitsorganisation und der beinahen Unmöglichkeit von Kommunikation über weite Distanzen hinweg. Die aktuell prekär Beschäftigten in den verschiedenen Sektoren mögen über den Globus hinweg weit verstreut sein, aber Smartphones, WhatsApp, Facebook und andere Internet-Tools erleichtern die Kommunikation untereinander entscheidend. Einzelne Beispiele der Organisation von Plattform-ArbeiterInnen etwa im Care- und im Reinigungs-Bereich (Beyond Care Cooperative, Si Se Puede, Coopify etc.) lassen hoffen, dass diese neuen Möglichkeiten in Zukunft vermehrt zur Organisation genützt werden.

\section{b) Eine eigene Klasse?}

Bevor wir uns der Frage der Organisierungsformen zuwenden, ist noch die Frage zu klären, ob es sich beim „Prekariat“ um eine eigene „Klasse“ handelt. Auf die Debatte rund um das Konzept von Standing und dessen politische Implikationen können wir an dieser Stelle nicht im Detail eingehen (Standing 2015a; Wright 2016; Paret 2016b; Braga 2016). Wir teilen die Kritik, die etwa von Wright vorgebracht wird, und sind wie er der Überzeugung, dass bei einer Gegenüberstellung von sich überschneidenden, geteilten und divergierenden Interessen im Hinblick auf verschiedene Handlungsebenen und Spielzüge die geteilten Interessen überwiegen und daher im Zusammenhang mit dem Surplus-Proletariat eher von einem vulnerablen Klassensegment als von einer eigenen, neuen „class in the making“ auszugehen wäre. Auch bei der Bewertung der Innovationsfähigkeit etablierter Gewerkschaften und der Handlungsfähigkeit von MigrantInnen (im Hinblick auf progressive politische Optionen) teilen wir die Kritik an den dichotomen Gegenüberstellungen von Standing, der einer Fraktion des „Prekariats“ (jüngere, formal höher gebildete urbane 
Personen mit einer Statusfrustration, die vor allem aus einer Nichtverwertbarkeit der Ausbildung resultiert) die Rolle einer neuen Avantgarde und divergierende Interessen gegenüber anderen Fraktionen des „Prekariats“ sowie anderen ArbeiterInnen zuschreibt. Dabei werden temporäre individuelle Positionen isoliert und fixiert. Gegen diese Betrachtungsweise spricht auf der individuellen Ebene der Wechsel zwischen verschiedenen Erwerbspositionen im Zeitverlauf (zur Persistenz prekärer Positionen gibt es bisher sehr wenig empirische Forschung; vgl. etwa zu Deutschland Allmendinger et al. 2018). Auf der Haushaltsebene zeigt beispielsweise Scully am Beispiel von Südafrika, dass formell und stabil Beschäftigte und prekär Erwerbstätige nicht in getrennten Welten, sondern im gleichen Haushalt wohnen und daher auf dieser Ebene sowohl Potenzial für Empathie und Interessenkonvergenzen zwischen diesen (temporären) Statuspositionen sichtbar wird.

\section{c) Konkrete Formierungs- und Organisierungs-} erfahrungen

$\mathrm{Ob}$ und wie sich Prekäre organisieren und mit anderen Gruppen kooperieren, ist daher eine empirische Frage, es ist vor dem Unterschied verschiedener politischer Gelegenheitsstrukturen eher von unterschiedlichen Formen von Kooperation und Konflikt mit anderen Akteuren wie Parteien, Gewerkschaften oder NGOs auszugehen als von einem klaren und eindeutigen Muster, das generalisiert werden könnte. ${ }^{24}$ Auch die auf den ersten Blick manchmal plausibel erscheinenden theoretischen Zuordnungen konkreter Aktionsformen zu einzelnen Gruppen (z. B. Streiks als Aktionsform organisierter ArbeiterInnen, Straßenproteste als Widerstandsform von Prekären oder des Lumpenproletariats) ist empirisch zu prüfen und ist nicht immer adäquat, wie etwa Nowak am Beispiel von Massenstreiks und Straßenprotesten in Indien und Brasilien zeigt (Nowak 2015).

24 Bonner/Spooner (2011: 100) verweisen z. B. auf unterschiedliche Gründe und Dynamiken im Zusammenhang mit der Entstehung von Gewerkschaften von informell Beschäftigten. Manche wurden von ehemals formell Beschäftigten und gewerkschaftlich Organisierten gegründet, andere von Gewerkschaften für formell Beschäftigte, wieder andere von Organisationen von Frauen, MigrantInnen oder verschiedenen NGOs initiiert und unterstützt etc. Diese unterschiedlichen „Vorgeschichten“ haben auch einen Einfluss auf die Organisationskultur, das Aktionsrepertoire und mögliche Bündnispartner.
Informell Erwerbstätige sind mit einer Reihe von Barrieren, wie z.B. räumlicher Isolation, einem Arbeitsplatz, der entweder mobil (auf der Straße) oder an entlegenen Orten ist, sehr langen und unregelmäßigen Arbeitszeiten, oft auch an verschiedenen Arbeitsorten bzw. in verschiedenen Jobs, mangelnden finanziellen Ressourcen, die für Mitgliederorganisationen ausschlaggebend sind, oder dem Mangel eines konkreten Gegenübers wie z.B. einer Unternehmensleitung konfrontiert, mit dem verhandelt werden könnte. StraßenverkäuferInnen, informelle TaxifahrerInnen oder MüllsammlerInnen stehen oft auch in unmittelbarer Konkurrenz zueinander. Viele sehen sich nicht als abhängig Beschäftigte oder haben mit Gewerkschaften schlechte Erfahrungen gemacht (Bonner/Spooner 2011). In den meisten Fällen finden sich daher nicht klassische Gewerkschaften wie bei formell Beschäftigten, sondern verschiedene Formen der Selbstorganisation und Interessenartikulation. Neben einigen erfolgreichen Beispielen regionaler, nationaler und transnationaler Organisierung sind auch viele gescheiterte Versuche zu beobachten, über die in der Literatur aber wesentlich seltener und weniger systematisch berichtet wird.

Als Initialzündung wird vielfach die Gründung der Self Employed Women's Association (SEWA) in Indien 1972 gesehen, die als erste Organisation von informell Erwerbstätigen gewerkschaftlich anerkannt wurde. Inzwischen repräsentiert SEWA über eine Million Frauen, betreibt rund 100 Kooperativen und bietet Kinderbetreuung und verschiedene Versicherungsleistungen an. In den 1980er-Jahren folgte die Organisierung von ArbeiterInnen in privaten Haushalten, StraßenhändlerInnen, MüllsammlerInnen, informellen TransportarbeiterInnen und HeimarbeiterInnen in Afrika, Lateinamerika und anderen Teilen Asiens, aus denen in den 1990er-Jahren Netzwerke wie StreetNet oder das International Domestic Workers' Network entstanden, das unter anderem ein Übereinkommen der ILO (Nr. 189, „Decent Work for Domestic Workers“) initiierte und durchsetzen konnte (Schurman/Eaton 2012). Inzwischen gibt es auch eine Reihe regionaler Netzwerke wie z. B. HomeNet South Asia oder HomeNet South-East Asia, die Allianzen von nationalen Netzwerken von HeimarbeiterInnen sind, die sich in ihrer jeweiligen Zusammensetzung (z. B. fast ausschließlich advokatorische NGOs in manchen Ländern, selbstorganisierte Mitgliederorganisationen in anderen) deutlich unterscheiden können (Bonner/ Spooner 2011: 95). Die Bewegung der Landlosen in Bra- 
silien (MST) oder Via Campesina, die Internationale der KleinbäuerInnen und LandarbeiterInnen haben im Zuge der globalisierungskritischen Protestmobilisierungen seit den späten 1990er-Jahren auch im globalen Norden einen gewissen Bekanntheitsgrad erreicht, für viele andere Initiativen und Organisationen im globalen Süden gilt das nicht in diesem Ausmaß.

Form und Dynamik der Organisierung von informell Erwerbstätigen hängen neben den verfügbaren Machtressourcen von politischen Gelegenheitsstrukturen ab. Als generelle Erfolgskriterien nennen z. B. Agarwala/Tilly (2015), dass nicht nur klassenbasiert, sondern intersektional mobilisiert wird, um schlagkräftige Organisationen aufzubauen, die wiederum verschiedene Allianzen mit anderen Akteuren ermöglichen. Der Erfolg von Worker Centers in den USA beruht z. B. wesentlich auf der Involvierung von Kirchen, Organisationen von ethnischen Gruppen und Gewerkschaften. Ein wichtiger Aspekt dürfte auch sein, dass der Erfolg derartiger Initiativen stärker als im Fall von Arbeitskämpfen vor allem von der „politischen Arena" abhängt bzw. in einer breiteren Öffentlichkeit verhandelt wird, weil arbeits- und sozialpolitische Aspekte in den meisten Fällen mit grundlegenden Fragen von citizenship und damit verbundenen Rechten verknüpft werden. In diesem Zusammenhang wird häufig auf das Konzept des Social Movement Unionism verwiesen, das im globalen Norden vor allem von Kim Moody und Peter Waterman popularisiert wurde. Das Konzept enthält deskriptive (die historische Verbindung von Arbeitskämpfen mit sozialen Bewegungen in einem autoritären Umfeld v. a. in Südafrika, Brasilien, den Philippinen und Südkorea) und normative bzw. präskriptive Aspekte. In der Debatte wird inzwischen häufiger auf Grenzen der Übertragbarkeit des Konzepts hingewiesen (Scipes 2014), der Grundgedanke der Öffnung, Verbreiterung, Demokratisierung und Radikalisierung über verstärkte gesellschaftliche Einbettung von Gewerkschaften wäre aber aus unserer Sicht auf jeden Fall weiterzuverfolgen. Anzustreben wären Aktions- und Organisationsformen, die über die enge, trade-unionistische Vertretung von Interessen von formell Beschäftigten einer Branche hinausgehen und die Interessen der gesamten ArbeiterInnenklasse hinsichtlich Arbeits- und Lebensbedingungen einbeziehen. Einen Weg zu finden, informell Erwerbstätige auf Augenhöhe einzubeziehen, wird für etablierte Gewerkschaften immer mehr zur Überlebensbedingung. Dabei ist allerdings auf jeweils unterschiedliche Kontexte zu achten, wenn Möglichkeiten und Gren- zen einzelner erfolgreicher Organisationsformen, wie z. B. Worker Centers (vgl. etwa Fine 2006; Milkman/ Ott 2014), diskutiert werden. Wie eine Untersuchung der Veränderung der Arbeitsorganisation und der Arbeitsbedingungen an verschiedenen Orten unter Berücksichtigung der Haushaltssituationen und des gesellschaftlichen Kontexts (verschiedene Formen von Privatisierung und Deregulierung) aussehen könnte, die es ermöglicht, Organisierungspotenziale auszuloten und $\mathrm{zu}$ kontextualisieren, haben beispielsweise Webster/Lambert/Bezuidenhout (2008) mit ihrem Buch Grounding Globalization. Labour in the Age of Insecurity gezeigt, in dem sie Gegenbewegungen in Australien, Südafrika und Südkorea vergleichen.

In den vergangenen Jahren wurden z. B. mit dem Global Labour Journal, dem Netzwerk WIEGO (Women in Informal Employment: Globalizing and Organizing), das eine Fülle von Informationen $\mathrm{zu}$ Arbeits- und Lebensbedingungen von informell Erwerbstätigen sowie den verschiedenen Formen der Organisierung dieser Gruppen aufbereitet hat (vgl. etwa die Datenbank WORD zur Organisierung von informell Erwerbstätigen http://www.wiego.org/wiegodatabase), oder dem ForscherInnennetzwerk EOIW (Experiences Organizing Informal Workers), das verschiedene mehr oder weniger erfolgreiche Organisierungsversuche sowie Voraussetzungen und Gründe für Erfolg oder Scheitern untersucht, schon sehr wichtige Schritte in Richtung „Emancipatory Global Labour Studies“ (Waterman 2012) unternommen, die es weiterzuverfolgen gilt. In den nächsten Jahren wird es darum gehen, aus der vorhandenen Fülle an Fallbeispielen zu mehr Systematisierung und hypothesengeleiteter Forschung zu gelangen (vgl. etwa Eaton et. al. 2017).

\section{Fazit}

Obwohl die Anzahl der LohnarbeiterInnen in den vergangenen 25 Jahren deutlich zugenommen hat, ist die unbefristete Vollzeit-Lohnarbeit im formellen Sektor global gesehen ein Minderheitenprogramm: Der überwiegende Teil der Menschen muss sich sein Einkommen aus allen erdenklichen Formen der prekären Beschäftigung (vulnerable, informell, Sklaven- und Kinderarbeit etc.) erwirtschaften. Das „Normalarbeitsverhältnis“ ist also nicht die Norm - weder in den Hoch-, noch in den Mittel- und Niedrigeinkommensländern -, die Norm sind vielmehr die prekären und informellen Formen! Beinahe die Hälfte aller Subalternen oder ca. 2 Mrd. Personen sind informell 
beschäftigt, in Afrika und Süd-/Südostasien gilt dies für beinahe alle Beschäftigungsverhältnisse.

Aus einer Makroperspektive betrachtet ist ein Mix an unterschiedlichen Erwerbs- und Einkommensformen (informelle Arbeit, Subsistenzwirtschaft, Lohnarbeit, Renteneinkommen) für die kapitalistische Profitmaximierung funktional, der jeweilige Mix ist von gesellschaftlichen Kräfteverhältnissen und technischen Möglichkeiten abhängig und regelmäßig Gegenstand von sozialen Auseinandersetzungen (Schmalz 2017). Aus einer Mikroperspektive betrachtet bedeutet die hier nur angedeutete analytische Dezentrierung der formellen Lohnarbeit auch einen verstärkten Fokus auf die Haushaltsebene, weil hier die unterschiedlichen Einkommensformen zusammenlaufen. Scully (2016) zeigt etwa anhand von Daten zu Südafrika, dass in den als „Formal Workers' Households“ klassifizierten Haushalten sehr unterschiedliche Kombinationen von verschiedenen Erwerbsstatus und Einkommensformen zu finden sind und mehr als die Hälfte (52 Prozent) aller als secure workers ausgewiesenen Personen in Haushalten lebt, in denen mindestens eine Person entweder arbeitslos oder informell beschäftigt ist oder eine Transferleistung bezieht (Scully 2016: 167).

Auch im Hinblick auf Formierungs- und Organisierungsprozesse der von uns untersuchten subalternen ArbeiterInnen ist zuerst einmal davon auszugehen, dass wir es nicht mit einer einzigen oder dominanten Form zu tun haben, sondern mit vielen unterschiedlichen Arten der Organisierung, von denen gewerkschaftliche Formen formell Beschäftigter oder Protestformen von Prekären im globalen Norden nur einzelne mögliche Varianten, nicht aber die Norm darstellen. Die vielköpfige Hydra (Linebaugh/Rediker) des „globalen Prekariats" kämpft und organisiert sich in vielen verschiedenen Formen, nicht nur in Form von Streiks oder Riots, sondern auch in anderen Formen der Selbstermächtigung.

Abschließend: Was bringt ein derart erweiterter Blick auf Informalisierung und Gegenbewegungen? Die Botschaft lautet jedenfalls nicht: Alles normal, relativ gesehen ist bei uns eh alles verhältnismäßig gut, jetzt gleichen sich die Verhältnisse bei uns halt wieder der globalen Normalität an. Die Erkenntnis könnte aus unserer Sicht eher lauten: In allen Weltregionen gibt es lange zurückreichende und vielfältige Erfahrungen im Kampf gegen Ausbeutung und Entrechtung, es wäre fahrlässig, nicht darauf zurückzugreifen und sich nicht zeitgerecht nach Beispielen für innovative Formen der Organisation und Kooperation umzuse- hen, die effektiven Widerstand gegen die fortschreitende Informalisierung ermöglichen können. Nicht nur die Informalisierung kommt wieder in die Zentren der Weltökonomie zurück, sondern auch verschiedene Traditionen und Erfahrungen der Organisierung der Subalternen. Erfahrungen mit Worker Centers in den USA (Fine 2006; Milkman/Ott 2014) zeigen, dass die Organisierung von und mit MigrantInnen dazu führen kann, dass das ursprünglich wesentlich breitere Aktionsrepertoire der ArbeiterInnenbewegung wieder erweitert wird. Auch für Österreich bleibt in diesem Zusammenhang zu hoffen, dass erfolgreiche innovative Initiativen wie beispielsweise UNDOK oder Sezonieri oder Organisierungsprozesse in der „Plattformökonomie“ (z. B. Foodora) Lernprozesse im Zusammenhang mit dem „remaking“ der ArbeiterInnenklasse vorantreiben.

\section{Literatur}

Agarwala, R./Tilly, Ch. (2015): Marx, Polanyi, and ... Informal Workers? Toward a New Social Contract for Workers. Online: http://futureswewant.net/agarwala-tilly-informalworkers/ [5. Oktober 2018]

Ahuja, R. (2013): Das Ähnliche speist den Unterschied: Die globale Wohlfahrtsdebatte und die Erzeugung „informeller Arbeit" im Indien des 20. Jahrhunderts. In: Burchardt, H.-J./Peters, S./Weinmann, N. (Hg.): Arbeit in globaler Perspektive. Facetten informeller Beschäftigung. Frankfurt am Main: Campus Verlag, 123-148.

Allmendinger, J./Jahn, K./ Promberger M./Schels, B./Stuth, St. (2018): Prekäre Beschäftigung und unsichere Haushaltslagen im Lebensverlauf: Gibt es in Deutschland ein verfestigtes Prekariat? WSI-Mitteilungen, 4, 259-269.

Altvater, E./Mahnkopf, B. (2002): Globalisierung der Unsicherheit. Arbeit im Schatten, Schmutziges Geld und informelle Politik. Münster: Westfälisches Dampfboot.

Anand, S./Segal, P. (2015): The Global Income Distribution. In: Bourguignon, F./Atkinson, A. (Hg.): Handbook of Income Distribution, Volume 2A. Elsevier B.V: North Holland, 937-979.

Artus, I. (2015): Prekär und widerständig. LuXemburg, 1, $40-47$.

Bescherer, P. (2013): Vom Lumpenproletariat zur Unterschicht. Produktivistische Theorie und politische Praxis. Frankfurt am Main: Campus Verlag.

Bonner, Ch./Spooner, D. (2011): Organizing in the Informal Economy: A Challenge for Trade Unions. Internationale Politik und Gesellschaft (IPG), 2, 87-105. 
Braga, R. (2016): On Standing's A Precariat Charter: Confronting the Precarisation of Labour in Brazil and Portugal. Global Labour Journal, 7 (2), 148-159.

Breman, J. (1976): A Dualistic Labour System? A Critique of the ,Informal Sector' Concept. Economic and Political Weekly, 11 (49), 1905-1908.

Britwum, A. O. (2018): Power Ressources and Organising Informal Economy Workers. Global Labour Journal, 9 (2), 249-253.

Burchardt, H.-J./Peters, St./Weinmann, N. (2013): Prekarität und Informalität - eine Annäherung in globaler Perspektive. In: Burchardt, H.-J./Peters, St./Weinmann, N. (Hg.): Arbeit in globaler Perspektive. Facetten informeller Beschäftigung. Frankfurt am Main: Campus Verlag, 9-28.

Candeias, M. (2007): Das „unmögliche“ Prekariat - Antwort auf Wacquant. Das Argument, 271, 410-423.

Ders. (2008): Genealogie des Prekariats. In: Altenhain, C./ Danilina, A./Hildebrandt, E./Kausch, St./Müller, A./ Roscher, T. (Hg.): Von „Neuer Unterschicht" und Prekariat. Gesellschaftliche Verhältnisse und Kategorien im Umbruch. Kritische Perspektiven auf aktuelle Debatten. Bielefeld: transcript, 121-138.

Candeias, M./Steckner, A. (2015): Prekär schreiten wir voran? Acht Thesen zu strategischen Problemen. LuXemburg, 1, 32-39.

Carstensen, A. L. (2014): Flexibilisierung und Prekarisierung von Arbeit in globalen Produktionsketten - informelle Heimarbeit in der Bekleidungsindustrie in Puebla und São Paulo. Sozial.Geschichte Online, 13, 7-40.

Castel, R. (2000): Die Metamorphosen der sozialen Frage. Eine Chronik der Lohnarbeit. Konstanz.

Castel, R./Dörre, K. (Hg.) (2009): Prekarität, Abstieg, Ausgrenzung. Die soziale Frage am Beginn des 21. Jahrhunderts. Frankfurt am Main: Campus Verlag.

Chen, M. A. (2012): The Informal Economy: Definitions, Theories and Policies. WIEGO Working Paper No 1. Online: http://www.wiego.org/sites/default/files/publications/ files/Chen_WIEGO_WP1.pdf [5. Oktober 2018].

Chen, M. A. (2013): Informalität, Geschlecht und die globalen Auswirkungen der großen Rezession. In: Burchardt, H.-J./Peters, St./Weinmann, N. (Hg.): Arbeit in globaler Perspektive. Facetten informeller Beschäftigung. Frankfurt am Main: Campus Verlag, 149-171.

Chun, J. J. (2009): Organizing at the Margins: The Symbolic Politics of Labor in South Korea and the United States. Ithaca/London: ILR Press.

Crane, B. (2017): The informal economy and India's working class. Uneven and combined development. International Socialist Review, \#106.

Davis, M. (2007): Planet der Slums. Berlin/Hamburg: Assoziation A.

Davies, J. B./Lluberas, R./Shorrocks, A. F. (2017): Estimating the level and distribution of global wealth, 2000-2014. Review of Income and Wealth, 63 (4), 731-759.
Denning, M. (2010): Wageless Life. New Left Review, 66, 79-97.

Dörre, K. (2013): Prekarität - ein System ständiger Bewährungsproben. In: Burchardt, H.-J./Peters, St./Weinmann, N. (Hg.): Arbeit in globaler Perspektive. Facetten informeller Beschäftigung. Frankfurt am Main: Campus Verlag, 29-54.

Eaton, A. E./Schurman, S. J./Chen, M. (Hg.) (2017): Informal Workers and Collective Action: A Global Perspective. Ithaca/London: ILR Press.

Eiden-Offe, P. (2017): Die Poesie der Klasse. Romantischer Antikapitalismus und die Erfindung des Proletariats. Berlin: Matthes \& Seitz.

EUROSTAT (2018): Underemployment and potential additional labour force statistics, 08/06/2018.

Fine, J. (2006): Worker Centers: Organizing Communities at the Edge of the Dream. Ithaca/London: ILR Press.

Fischer, K. (2017): Globale Einkommensverteilung: Vom Glück der Geburt. In: Dimmel, N./Hofmann, J./Schenk, M./Schürz, M. (Hg.): Handbuch Reichtum. Innsbruck: Studien Verlag, 34-50.

Foster, J. B./McChesney, R. W./Jonna, R. J. (2011): The Global Reserve Army of Labor and the New Imperialism. Monthly Review, 63 (6).

Foster, J. B./Jonna, R.J. (2016): Marx's Theory of Working Class Precariousness. Monthly Review, 67 (11).

Gallup (2013): State of the Global Workplace. Employee Engagement Insights for Business Leaders Worldwide.

Hart, K. (1973): Informal Income Opportunities and Urban Employment in Ghana. Journal of Modern African Studies, 11 (1), 61-89.

ILO (1972): Employment, Incomes and Equality: A Strategy for Increasing Productive Employment in Kenya. Research Paper.

ILO (2008): Beyond Unemployment: Measurement of other forms of Labour Underutilization. Room document for the 18th International Conference of Labour Statisticians Geneva, 24 November-5 December 2008.

ILO (2013): Resolution concerning statistics of work, employment and labour underutilization. Online: https://www. ilo.org/wcmsp5/groups/public/---dgreports/---stat/documents/normativeinstrument/wcms_230304.pdf [15. April 2018].

ILO (2016a): World Employment and Social Outlook. Trends 2016. Geneva.

ILO (2016b): Non-Standard Employment Around the World: Understanding challenges, shaping prospects. Report. Geneva.

ILO (2017a): Global Estimates of Child Labour: Results and trends, 2012-2016. Geneva.

ILO (2017b): Global Estimates of Modern Slavery: Forced labour and forced marriage. Geneva.

ILO (2017c), World Social Protection Report 2017-2019: Universal social protection to achieve the Sustainable Development Goals. Report. Geneva. 
ILO (2018a): Women and Men in the Informal Economy. A Statistical Picture. Report. Geneva.

ILO (2018b): World Employment Social Outlook. Trends 2018. Geneva.

ILO-WESO: World Employment and Social Outlook, Data Finder. Online: http://www. ilo.org/wesodata/ [28. April 2018].

ILOSTAT. Online: http://www.ilo.org/ilostat/ [28. April 2018].

Kapsos, St./Bourmpoula, E. (2013): Employment and economic classes in the developing world. ILO Research Paper No 6, June 2013.

Kössler, R. (2016): Lohnnexus, Prekariat, globale Arbeiterklasse und soziale Kämpfe. In: Wemheuer, F. (Hg.): Marx und der globale Süden. Köln: PapyRossa, 298-323.

Komlosy, A./Parnreiter, Ch./Stacher, I. (Hg.): Ungeregelt und unterbezahlt. Der informelle Sektor in der Weltwirtschaft. Frankfurt am Main: Brandes \& Aspel.

Lakner, Ch./Milanovic, B. (2015): Global Income Distribution: From the Fall ot the Berlin Wall to the Great Recession. The World Bank Economic Review, 30 (2), 1-30.

Langthaler, H. (1997): Globalisierung der Konzernstrategien und Informalisierung. Das Beispiel der Textilindustrie. In: Komlosy, A./Parnreiter, Ch./Stacher, I./Zimmermann, S. (Hg.): Ungeregelt und unterbezahlt. Der informelle Sektor in der Weltwirtschaft. Frankfurt am Main: Brandes \& Apsel, 221-234.

Lee, Ch. K./Kofman, Y. (2012): The Politics of Precarity: Views Beyond the United States. Work and Occupations, 39 (4), 388-408.

Lindell, I. (2010): Between Exit and Voice: Informality and the Spaces of Popular Agency. African Studies Quarterly, 11 (2\&3), 1-11.

Linebaugh, P./Rediker, M. (2008): Die vielköpfige Hydra. Die verborgene Geschichte des revolutionären Atlantiks. Berlin/Hamburg: Assoziation A.

Marx, K. (1989), Das Kapital, Bd 1. Dietz Verlag Berlin.

Mayer-Ahuja, N. (2013): Prekär, informell - weiblich? Zur Bedeutung von „Gender“ für die Aushöhlung arbeitspolitischer Standards. In: Burchardt, H.-J./Peters, St./Weinmann, N. (Hg.): Arbeit in globaler Perspektive. Facetten informeller Beschäftigung. Frankfurt am Main: Campus Verlag, 55-78.

Mayer-Ahuja, N. (2017): Die Globalität unsicherer Arbeit als konzeptionelle Provokation. Geschichte und Gesellschaft, 43 (2), 264-296.

Milanovic, B. (2012): Global Inequality: From Class to Location, from Proletarians to Migrants, Global Policy, 3 (2), 125-134.

Milkman, R./Ott, E. (Hg.) (2014): New Labor in New York: Precarious Workers and the Future of the Labor Movement. Ithaca/London: ILR Press.

Mosoetsa, S./Stillerman, J./Tilly, Ch. (2016): Precarious Labor, South and North: An Introduction. International Labor and Working Class History, No. 89, Special Issue on Precarious Labor in Global Perspective, 1-15.
Munck, R. (2013): The Precariat: a view from the South. Third World Quarterly, 34 (5), 747-762.

Nowak, J. (2015): Massenstreiks und Straßenproteste in Indien und Brasilien. PERIPHERIE 137, 74-102.

OECD (2010): Tackling Inequalities in Brazil, China, India and South Afrika. Paris.

Paret, M. (2016a): Politics of Solidarity and Agency in an Age of Precarity. Global Labour Journal, 7 (2), 174-188.

Paret, M. (2016b): Towards a Precarity Agenda. Global Labour Journal, 7 (2), 111-122.

Parnreiter, Ch. (1997): Die Renaissance der Ungesichertheit: Über die Ausweitung informeller Beziehungen zwischen Kapital und Arbeit im Zeitalter der Globalisierung. In: Komlosy, A./Parnreiter, Ch./Stacher, I./Zimmermann, S. (Hg.): Ungeregelt und unterbezahlt. Der informelle Sektor in der Weltwirtschaft. Frankfurt am Main: Brandes \& Apsel, 203-220.

Roser, M. (2016): Global Economic Inequality. Online: https:// ourworldindata.org/global-economic-inequality [28. April 2018].

Sassen, S. (1997): Informalisierung in den Global Cities der hochentwickelten Marktwirtschaften: hausgemacht oder importiert? In: Komlosy, A./Parnreiter, Ch./Stacher, I./ Zimmermann, S. (Hg.): Ungeregelt und unterbezahlt. Der informelle Sektor in der Weltwirtschaft. Frankfurt am Main: Brandes \& Apsel, 235-248.

Savage, M. (2015): Social Class in the 21st Century. London: Penguin Books.

Schmalz, S. (2017): Globale Ausbeutung der Ware Arbeitskraft. In: Dimmel, N./Hofmann, J./Schenk, M./Schürz, M. (Hg.): Handbuch Reichtum. Innsbruck: Studien Verlag, 283-294.

Schmalz, S./Ludwig, C./Webster, E. (2019): Power Resources and Global Capitalism. Global Labour Journal, 10 (1), 84-90.

Schurman, S. J./Eaton, A. E. (2012): Trade Union Organizing in the Informal Economy: A Review of the Literature on Organizing in Africa, Asia, Latin America, North America and Western, Central and Eastern Europe. Report to the American Center for International Labor Solidarity.

Scully, B. (2016): Precarity North and South: A Southern Critique of Guy Standing. Global Labour Journal, 7 (2), 160-173.

Selwyn, B. (2014): Beyond 2015: Is Another Development Possible? Online: https://socialistproject.ca/2014/o9/b104o/ [10. Juli 2018].

Scipes, K. (2014): Social Movement Unionism or Social Justice Unionism? Disentangling Theoretical Confusion within the Global Labor Movement. Class, Race and Corporate Power, 2 (3), Article 9.

Silver, B. (2005): Forces of Labor. Arbeiterbewegungen und Globalisierung seit 1870. Berlin/Hamburg: Assoziation A.

Srivastava, R. (2016): Structural Change and non-standard forms of employment in India. ILO Conditions of work and employment series 68 . 
Srnicek, N./Williams, A. (2015): Die Zukunft erfinden. Postkapitalismus und eine Welt ohne Arbeit. Berlin: Edition Tiamat.

Standing, G. (2015a): The Precariat and Class Struggle. Revista Critica de Ciencias Sociais Annual Review, 7, 3-16.

Ders. (2015b): Prekariat: Die neue explosive Klasse. Münster: Unrast Verlag.

Ders. (2016a): Eine Charta des Prekariats: Von der ausgeschlossenen zur gestaltenden Klasse. Münster: Unrast Verlag.

Ders. (2016b): The Precariat, Class and Progressive Politics: A Response. Global Labour Journal, 7 (2), 189-20o.

Thompson, E. P. (1966): The making of the English working class. New York.

Van der Linden, M. (2010): Plädoyer für eine historische Neubestimmung der Welt-Arbeiterklasse. In: Thien, H.G. (Hg.): Klassen im Postfordismus. Münster: Westfälisches Dampfboot, 357-378.

Ders. (2016): Global Labour: A Not-so-grand Finale and Perhaps a New Beginning. Global Labour Journal, 7 (2), 201-210.

Ders. (2017a): Workers of the World. Eine Globalgeschichte der Arbeit. Frankfurt/Main: Campus.

Ders. (2017b): Die Arbeiterklasse wächst und bewegt sich. Neues Deutschland, 11.07.2017.

Waterman, P. (2012): An Emancipatory Global Labour Studies is Necessary! On Rethinking the Global Labour Movement in the Hour of Furnaces. IISH Research Paper 49/2012.

Webster, E./Lambert, R./Bezuidenhout, A. (2008): Grounding Globalization. Labour in the Age of Insecurity. Oxford: Blackwell.

Webster, E. (2015): Labour after Globalisation: Old and New Sources of Power. ISER Working Paper.

Wright, E. O. (2016): Is the Precariat a Class? Global Labour Journal, 7 (2), 123-135. 